ESAIM: COCV 20 (2014) 1224-1248

DOI: $10.1051 / \mathrm{cocv} / 2014014$
ESAIM: Control, Optimisation and Calculus of Variations

www.esaim-cocv.org

\title{
HÖLDER EQUIVALENCE OF THE VALUE FUNCTION FOR CONTROL-AFFINE SYSTEMS *
}

\author{
DARIO PRANDI ${ }^{1,2,3}$
}

\begin{abstract}
We prove the continuity and the Hölder equivalence w.r.t. an Euclidean distance of the value function associated with the $\mathrm{L}^{1}$ cost of the control-affine system $\dot{q}=f_{0}(q)+\sum_{j=1}^{m} u_{j} f_{j}(q)$, satisfying the strong Hörmander condition. This is done by proving a result in the same spirit as the Ball-Box theorem for driftless (or sub-Riemannian) systems. The techniques used are based on a reduction of the control-affine system to a linear but time-dependent one, for which we are able to define a generalization of the nilpotent approximation and through which we derive estimates for the shape of the reachable sets. Finally, we also prove the continuity of the value function associated with the $\mathrm{L}^{1}$ cost of time-dependent systems of the form $\dot{q}=\sum_{j=1}^{m} u_{j} f_{j}^{t}(q)$.
\end{abstract}

Mathematics Subject Classification. 53C17, 93C15.

Received April 24, 2013. Revised November 22, 2013.

Published online August 8, 2014.

\section{INTRODUCTION}

A control-affine system on a smooth manifold $M$ is a control system in the form

$$
\dot{\gamma}(t)=f_{0}(\gamma(t))+\sum_{i=1}^{m} u_{i}(t) f_{i}(\gamma(t)), \quad \text { a.e. } t \in[0, T],
$$

where $u:[0, T] \rightarrow \mathbb{R}^{m}$ is an integrable control function and $f_{0}, f_{1}, \ldots, f_{m}$ are (not necessarily linearly independent) smooth vector fields. The uncontrolled vector field $f_{0}$ is called the drift. These kind of systems appear in plenty of applications. As examples we cite: mechanical systems with controls on the acceleration (see e.g., $[7,14]$ ), where the drift is the velocity, quantum control (see e.g., $[11,17]$ ), where the drift is the free Hamiltonian, or the swimming of microscopic organisms (see e.g., [33]). We always assume the strong Hörmander

Keywords and phrases. Control-affine systems, time-dependent systems, sub-Riemannian geometry, value function, Ball-Box theorem, nilpotent approximation.

* This work was supported by the European Research Council, ERC StG 2009 "GeCoMethods", Contract No. 239748, by the ANR project GCM, program "Blanche", Project No. NT09_504490, and by the Laboratoire d'Excellence Archimède, AixMarseille Université.

1 LSIS, Université de Toulon, 83957 La Garde cedex, France. dario.prandi@univ-tln.fr

2 Centre National de Recherche Scientifique (CNRS), CMAP, École Polytechnique, Route de Saclay, 91128 Palaiseau cedex, France.

3 Team GECO, INRIA-Centre de Recherche Saclay, France. 
condition, i.e., that the iterated Lie brackets of the controlled vector fields $f_{1}, \ldots, f_{m}$ generate the whole tangent space at any point. This guarantees the small time local controllability of system (1.1) and allows us to associate to (1.1) a sub-Riemannian control system. Such assumption is generically satisfied, e.g., by finite-dimensional quantum control systems with two controls, as the ones studied in $[12,13,18]$. Except when explicitly stated, we do not make any assumption on the dimension of $\operatorname{span}\left\{f_{1}(q), \ldots, f_{m}(q)\right\}$ which, in particular, can depend on the point $q \in M$.

Let the cost of a curve $\gamma$ solving (1.1) be $c_{f_{0}}(\gamma)=\min \|u\|_{\mathrm{L}^{1}}$, where the minimum is taken over all the possible $u(\cdot)$ satisfying the above ODE. Due to the presence of the drift, the cost depends on the parametrization of the curve. Through this cost we can define the value function between $q, q^{\prime} \in M$ of the control system at time $T>0$ as

$$
\rho_{T}^{f_{0}}\left(q, q^{\prime}\right)=\inf \left\{c_{f_{0}}(\gamma): \gamma:\left[0, T^{\prime}\right] \rightarrow M \text { solves }(1.1), \gamma(0)=q, \gamma\left(T^{\prime}\right)=q^{\prime} \text {, and } T^{\prime} \leq T\right\} .
$$

We remark that, thanks to the strong Hörmander condition, such value function is always finite (see e.g., [29]). Moreover, since $f_{1}, \ldots, f_{m}$ are not assumed to be linearly independent, system (1.1) could be redundant, in the sense that it could be possible to discard some control vector fields without changing the value function.

The literature on control systems in the form (1.1) is rich. A lot of work has been devoted to develop sufficient conditions for small time local controllability under more general assumptions than the strong Hörmander condition, e.g., $[9,16,36]$. Moreover, the regularity of the value function for $\mathrm{L}^{p} \operatorname{costs}, p>1$, or for the minimaltime problem with bounded controls has been deeply studied, e.g., $[3,10,15,21,35,37,38]$. In particular it is well-known (see $[3,38])$ that $\mathrm{L}^{p}$ costs with $p>1$ give rise to value functions that in general are not even continuous. On the other hand, very little is known for the $\mathrm{L}^{1}$ cost, which is a natural choice when looking at system (1.1) as a perturbation of a sub-Riemannian system (i.e., a driftless control-affine system). In this paper we focus on the regularity of the value function $\rho_{T}^{f_{0}}(\cdot, \cdot)$ associated with such cost, proving that it is continuous and, in coordinates, Hölder equivalent to the Euclidean distance from $\mathrm{e}^{[0, T] f_{0}}(q)$.

\subsection{Sub-Riemannian control systems}

When posing $f_{0}=0$ in (1.1) we obtain the sub-Riemannian control system associated with (1.1), i.e., the driftless control system in the form

$$
\dot{\gamma}(t)=\sum_{i=1}^{m} u_{i}(t) f_{i}(\gamma(t)), \quad \text { a.e. } t \in[0, T],
$$

such that the following is satisfied.

(SR1) The family of smooth vector fields $\left\{f_{1}, \ldots, f_{m}\right\}$ satisfies the Hörmander condition, i.e., its iterated Lie brackets generate the whole tangent space at any point.

The length of a curve $\gamma$ solving (1.2), is defined as length $(\gamma)=\min \|u\|_{\mathrm{L}^{1}}$. It is a length since, by the linearity of the system w.r.t. $u$, it is independent of the parametrization of $\gamma$. Finally, we define

$$
d_{\mathrm{SR}}\left(q, q^{\prime}\right)=\inf \left\{\operatorname{length}(\gamma): \gamma:[0,1] \rightarrow M \text { is a solution of }(1.2), \gamma(0)=q \text { and } \gamma(1)=q^{\prime}\right\} .
$$

By the Hörmander condition, $d_{\mathrm{SR}}$ is a distance, called Carnot-Carathéodory distance, endowing $M$ with a natural metric space structure. A manifold considered together with a sub-Riemannian control system is called a sub-Riemannian manifold.

A fundamental result in the theory of sub-Riemannian manifolds is the celebrated Ball-Box theorem (see for example [8]). This theorem gives a rough description of the infinitesimal shape of the sub-Riemannian balls. Namely, define $\Delta^{1}=\operatorname{span}\left\{f_{1}, \ldots, f_{m}\right\}$ and $\Delta^{s+1}=\Delta^{s}+\left[\Delta^{s}, \Delta^{1}\right]$, for every $s \in \mathbb{N}$. By the Hörmander condition, for each $q \in M$ there exists a (minimal) $r \in \mathbb{N}$ such that $\Delta^{r}(q)=T_{q} M$, which is called the degree of non-holonomy 
of the sub-Riemannian control system at $q$. Then, the Ball-Box theorem states that the sub-Riemannian ball at a point $q \in M$ of small radius $\varepsilon$ is equivalent, in privileged coordinates, to the box

$$
\underbrace{[-\varepsilon, \varepsilon] \times \ldots \times[-\varepsilon, \varepsilon]}_{\operatorname{dim} \Delta^{1}(q)} \times \ldots \times \underbrace{\left[-\varepsilon^{s}, \varepsilon^{s}\right] \times \ldots \times\left[-\varepsilon^{s}, \varepsilon^{s}\right]}_{\operatorname{dim} \Delta^{s}(q)-\operatorname{dim} \Delta^{s-1}(q)} \times \ldots \times \underbrace{\left[-\varepsilon^{r}, \varepsilon^{r}\right] \times \ldots \times\left[-\varepsilon^{r}, \varepsilon^{r}\right]}_{\operatorname{dim} \Delta^{r}(q)-\operatorname{dim} \Delta^{r-1}(q)} .
$$

By this we mean that, for some constant $C>0$, the sub-Riemannian ball is contained in a box of side $C \varepsilon$, and contains a box of side $\varepsilon / C$.

This fact has a plethora of applications. The most important for our purposes is that it allows to prove the Hölder equivalence in coordinates between the sub-Riemannian distance and the Euclidean distance, namely that, for $q^{\prime}$ sufficiently close to $q$, it holds

$$
\left|q^{\prime}-q\right| \lesssim d_{\mathrm{SR}}\left(q, q^{\prime}\right) \lesssim\left|q^{\prime}-q\right|^{1 / r}
$$

Here, we use " $\lesssim$ " to denote an inequality up to a multiplicative constant, independent of $q^{\prime}$. Then, among many others, the Ball-Box theorem is a fundamental step in the computation of the Hausdorff dimension of the manifold (see [30]), and it is used to obtain asymptotic estimates on the heat kernel (see e.g., $[2,20,28,32])$. Moreover, it is the main tool in computing the asymptotic equivalents of the entropy and the complexity of curves (see e.g., $[22,23,25,27,31]$ ).

\subsection{Main results}

We now state the main result of the paper, a generalization of (1.3) in the context of control-affine systems. Let us fix a point $q \in M$ and make the following assumptions.

(D1) The family $\left\{f_{0}, f_{1}, \ldots, f_{m}\right\}$ satisfies the strong Hörmander condition, i.e., the family $\left\{f_{1}, \ldots, f_{m}\right\}$ satisfies the Hörmander condition and thus defines a sub-Riemannian control system.

(D2) The point $q$ is regular for the integral curve of the drift, i.e., is such that $\operatorname{dim} \Delta^{s}\left(\mathrm{e}^{t f_{0}}(q)\right), s \in \mathbb{N}$, is constant for small $t$.

(D3) The point $q$ is regular w.r.t. the drift, in the sense that there exists $s \in \mathbb{N}$ such that $f_{0}\left(q^{\prime}\right) \in \Delta^{s}\left(q^{\prime}\right) \backslash$ $\Delta^{s-1}\left(q^{\prime}\right)$, for any $q^{\prime}$ near $q$.

Assumption (D1) is crucial to be able to exploit the underlying sub-Riemannian system, while (D2) is needed in order for the sub-Riemannian Ball-Box theorem to hold uniformly near $q$ along the integral curve of the drift. Although (D3) seems fairly strong, we remark that it is always satisfied at generic points (i.e., on an open and dense subset of $M)$.

Theorem 1.1. Let (D1) be satisfied and let $q \in M$ be a point satisfying (D2) and (D3). Let $z=\left(z_{1}, \ldots, z_{n}\right)$ be a system of privileged coordinates at $q$ for $\left\{f_{1}, \ldots, f_{m}\right\}$, rectifying $f_{0}$ as the $k$-th coordinate vector field $\partial_{z_{k}}$, for some $1 \leq k \leq n$. Then, for sufficiently small $T$ and $\varepsilon$, if $\rho_{T}^{f_{0}}\left(q, q^{\prime}\right) \leq \varepsilon$, in $z$ coordinates it holds

$$
\operatorname{dist}\left(z\left(q^{\prime}\right), z\left(\mathrm{e}^{[0, T] f_{0}} q\right)\right) \lesssim \rho_{T}^{f_{0}}\left(q, q^{\prime}\right) \lesssim \operatorname{dist}\left(z\left(q^{\prime}\right), z\left(\mathrm{e}^{[0, T] f_{0}} q\right)\right)^{1 / r}
$$

Here for any $x \in \mathbb{R}^{n}$ and $A \subset \mathbb{R}^{n}$, $\operatorname{dist}(x, A)=\inf _{y \in A}|x-y|$ denotes the Euclidean distance between them, $\mathrm{e}^{t f_{0}}$ denotes the flow of $f_{0}$, and $r$ is the degree of non-holonomy of the sub-Riemannian control system defined by $\left\{f_{1}, \ldots, f_{m}\right\}$.

In this result, instead of the Euclidean distance from the origin that appeared in (1.3), we have the distance from the integral curve of the drift. This is due to the fact that moving in this direction has null cost. We remark also that, under (D3), it is always possible to build a system of privileged coordinates rectifying the drift (see Prop. 2.6 and the discussion before). 
As in the sub-Riemannian case, Theorem 1.1 is a consequence of an estimate on the shape of the reachable sets, contained in Theorem 4.3. Moreover, although Theorem 1.1 seems a natural generalization of (1.3), the shape of the reachable sets described in Theorem 4.3 is much more complicated than the boxes of the sub-Riemannian case, yielding a more difficult proof. We remark that it is possible to remove (D2), using the uniform subRiemannian estimates contained in [26], but at the cost of an increasead complexity in the description of the reachable sets. Theorems 1.1 and 4.3 represent the key step for generalizing the estimates on the complexity of curves from sub-Riemannian control systems to control-affine systems.

It is worth to mention that these results regarding control-affine systems are obtained by reducing them, as in [3], to time-dependent control systems in the form

$$
\dot{\gamma}(t)=\sum_{i=1}^{m} u_{i}(t) f_{i}^{t}(\gamma(t)), \quad \text { a.e. } t \in[0, T],
$$

where $f_{i}^{t}=\left(\mathrm{e}^{-t f_{0}}\right)_{*} f_{i}$ is the pull-back of $f_{i}$ through the flow of the drift. On these systems, that are linear in the control, we are able to define a good notion of approximation of the control vector fields. Namely, in Section 3.3 we will define a generalization of the nilpotent approximation, used in the sub-Riemannian context, taking into account the fact that in system (1.4), exploiting the time, we can generate the direction of the brackets between $f_{0}$ and the $f_{j}$ s. This approximation and an iterated integral method yield the correct estimates on the reachable set of system (1.4) contained in Theorem 3.9, where we do not need to assume (D2) nor (D3). Adding these assumptions and applying the estimates of Theorem 3.9, we then derive Theorem 4.3.

\subsection{Structure of the paper}

The paper is divided in three sections. In Section 2 we recall some preliminaries on sub-Riemannian control systems, used in the following sections. In Section 3 we consider control systems in the form (1.4), and we prove the continuity of the value function for general time-dependent vector fields, under the following assumptions.

(T1) The map $t \mapsto f_{i}^{t}$ is smooth for $1 \leq i \leq m$ and $t \in I$.

(T2) The family of smooth vector fields $\left\{f_{1}^{t}, \ldots, f_{m}^{t}\right\}$ satisfies the strong Hörmander condition, i.e., for any $t$ the family $\left\{f_{1}^{t}, \ldots, f_{m}^{t}\right\}$ satisfies the Hörmander condition.

Then, in Theorem 3.9, restricting to the case where the time dependency is explicitly given as $f_{i}^{t}=\left(\mathrm{e}^{-t f_{0}}\right)_{*} f_{i}$, we establish some estimates on the reachable sets, in the same spirit as the Ball-Box theorem. Finally, in Section 4 we consider control-affine systems. Here, after proving the relation between control-affine systems and time-dependent systems, we prove the continuity of the value function. Then, in Lemma 4.5, exploiting the affine nature of the control system, we give an upper bound on the time needed to join two points $q$ and $q^{\prime}$ as a function of $\rho_{T}^{f_{0}}\left(q, q^{\prime}\right)$. From this fact and the estimates of Section 3 we finally obtain Theorems 1.1 and 4.3.

\section{Preliminaries on sub-Riemannian Geometry}

Throughout this paper, $M$ is an $n$-dimensional connected smooth manifold. In this section we recall some classical notions and results of sub-Riemannian geometry.

\subsection{Sub-Riemannian control systems}

A sub-Riemannian (or non-holonomic) control system on $M$ is a control system in the form

$$
\dot{q}=\sum_{i=1}^{m} u_{i} f_{i}(q), \quad q \in M, \quad u=\left(u_{1}, \ldots, u_{m}\right) \in \mathbb{R}^{m},
$$

where $\left\{f_{1}, \ldots, f_{m}\right\}$ is a family of smooth vector fields on $M$. We let $f_{u}=\sum_{i=1}^{m} u_{i} f_{i}$. Moreover, as already stated in the introduction, we will assume the following. 
(SR1) The family of smooth vector fields $\left\{f_{1}, \ldots, f_{m}\right\}$ satisfies the Hörmander condition, i.e., its iterated Lie brackets generate the whole tangent space at any point.

An absolutely continuous curve $\gamma:[0, T] \rightarrow M$ is (SR)-admissible if there exists a control $u \in \mathrm{L}^{1}\left([0, T], \mathbb{R}^{m}\right)$ such that $\dot{\gamma}(t)=f_{u(t)}(\gamma(t))$, for a.e. $t \in[0, T]$. The curve is said to be associated to any such control. The length of $\gamma$ is defined as

$$
\operatorname{length}(\gamma)=\min \|u\|_{\mathrm{L}^{1}\left([0, T], \mathbb{R}^{m}\right)},
$$

where the minimum is taken over all controls $u$ such that $\gamma$ is associated with $u$. It is attained, due to convexity reasons. Notice that, by definition, length $(\gamma)$ is invariant under time reparametrization of the curve. The distance induced by the sub-Riemannian system on $M$ is then defined as

$$
d_{\mathrm{SR}}\left(q, q^{\prime}\right)=\inf \left\{\operatorname{length}(\gamma): \gamma(\mathrm{SR}) \text {-admissible and } \gamma: q \rightsquigarrow q^{\prime}\right\}
$$

where $\gamma: q \rightsquigarrow q^{\prime}$ stands for $\gamma:[0, T] \rightarrow M$, for some $T>0, \gamma(0)=q$ and $\gamma(T)=q^{\prime}$.

Let $\Delta$ be the $\mathrm{C}^{\infty}$-module generated by the vector fields $\left\{f_{1}, \ldots, f_{m}\right\}$ (in particular, it is closed under multiplication by $\mathrm{C}^{\infty}(M)$ functions and summation). Let $\Delta^{1}=\Delta$, and define recursively $\Delta^{s+1}=\Delta^{s}+\left[\Delta^{s}, \Delta\right]$, for every $s \in \mathbb{N}$. Due to the Jacobi identity $\Delta^{s}$ is the $\mathrm{C}^{\infty}$-module of linear combinations of all commutators of $f_{1}, \ldots, f_{m}$ with length $\leq s$. For $q \in M$, let $\Delta^{s}(q)=\left\{f(q): f \in \Delta^{s}\right\} \subset T_{q} M$. Assumption (SR1), i.e. the Hörmander condtion, can then be expressed as $\bigcup_{s \geq 1} \Delta^{s}(q)=T_{q} M$ for any $q \in M$.

By the Chow-Rashevsky theorem (see for instance [1]), the hypothesis of connectedness of $M$ and the Hörmander condition guarantee the finiteness and continuity of $d_{\mathrm{SR}}$ with respect to the topology of $M$. Hence, the function $d_{\mathrm{SR}}$, called sub-Riemannian or Carnot-Carathéodory distance, induces on $M$ a metric space structure. The open balls of radius $\varepsilon>0$ and centered at $q \in M$ with respect to $d_{\mathrm{SR}}$ are denoted by $B_{\mathrm{SR}}(q, \varepsilon)$.

We say that a (SR)-admissible curve $\gamma$ is a minimizer of the sub-Riemannian distance between $q, q^{\prime} \in M$ if $\gamma: q \rightsquigarrow q^{\prime}$ and length $(\gamma)=d_{\mathrm{SR}}\left(q, q^{\prime}\right)$. Equivalently, $\gamma$ is a minimizer between $q, q^{\prime} \in M$ if it is a solution of the free-time optimal control problem, associated with (SR),

$$
\|u\|_{\mathrm{L}^{1}(0, T)}=\int_{0}^{T} \sqrt{\sum_{j=1}^{m} u_{j}^{2}(t)} \mathrm{d} t \rightarrow \min , \quad \gamma(0)=q, \quad \gamma(T)=q^{\prime}, \quad T>0 .
$$

Indeed, the sub-Riemannian distance is the value function associated with this problem. It is a classical result that, for any couple of points $q, q^{\prime} \in M$ sufficiently close, there exists at least one minimizer.

Remark 2.1. The optimal control problem (2.2) is equivalent to the following, with $p \geq 1$ and $T>0$ fixed,

$$
\|u\|_{L^{p}(0, T)}=\left(\int_{0}^{T}|u|^{p} \mathrm{~d} t\right)^{1 / p} \rightarrow \min , \quad \gamma(0)=q, \quad \gamma(T)=q^{\prime} .
$$

In fact, due to the invariance under time reparametrization of system (SR), in (2.2) we can fix either $T>0$ or the Euclidean norm of $u$. Moreover, by the Hölder inequality, for any $p>1$, letting $p^{\prime}$ be the conjugated exponent to $p\left(\right.$ i.e., $\left.1 / p+1 / p^{\prime}=1\right)$, we get $\|u\|_{\mathrm{L}^{1}(0, T)} \leq T^{1 / p^{\prime}}\|u\|_{L^{p}(0, T)}$, with the equality holding if and only if $|u|$ is constant. From these two facts, it is easy to check that minimizers of the optimal control problem (2.3) coincide with the minimizers of (2.2) with constant norm. Thus the two optimal control problems are equivalent.

A similar reasoning allows to show that $(2.2)$ is equivalent to the minimum time optimal control problem with bounded controls, i.e., fixing $C>0$,

$$
T \rightarrow \min , \quad|u| \leq C, \quad \gamma(0)=q, \quad \gamma(T)=q^{\prime} .
$$


Remark 2.2. This control theoretical setting can be stated in purely geometric terms. Indeed, it is equivalent to a generalized sub-Riemannian structure. Such a structure is defined by a rank-varying smooth distribution and a Riemannian metric on it (see [1] for a precise definition). In a sub-Riemannian control system, in fact, the map $q \mapsto \operatorname{span}\left\{f_{1}(q), \ldots, f_{m}(q)\right\} \subset T_{q} M$ defines a rank-varying smooth distribution, which is naturally endowed with the Riemannian norm defined, for $v \in \Delta(q)$, by

$$
\mathbf{g}(q, v)=\inf \left\{|u|=\sqrt{u_{1}^{2}+\cdots+u_{m}^{2}}: f_{u}(q)=v\right\} .
$$

The pair $(\Delta, \mathbf{g})$ is thus a generalized sub-Riemannian structure on $M$. Conversely, every rank-varying distribution is finitely generated, see $[1,4,6,19]$, and thus a sub-Riemannian distance can be written, globally, as the value function of a control system of the type (SR).

\subsection{Privileged coordinates and nilpotent approximation}

We now introduce the equivalent, in the sub-Riemannian context, of the linearization of a vector field. This classical procedure, called nilpotent approximation, is possible only in carefully chosen sets of coordinates, called privileged coordinates.

Since by (SR1) the family $\left\{f_{1}, \ldots, f_{m}\right\}$ satisfies the Hörmander condition, the values of the sets $\Delta^{s}$ at $q$ form a flag of subspaces of $T_{q} M$,

$$
\Delta^{1}(q) \subset \Delta^{2}(q) \subset \ldots \subset \Delta^{r}(q)=T_{q} M .
$$

The integer $r=r(q)$, which is the minimum number of brackets required to recover the whole $T_{q} M$, is called degree of non-holonomy (or step) of the family $\left\{f_{1}, \ldots, f_{m}\right\}$ at $q$. Set $n_{s}(q)=\operatorname{dim} \Delta^{s}(q)$. The integer list $\left(n_{1}(q), \ldots, n_{r}(q)\right)$ is called the growth vector at $q$. From now on we fix $q \in M$, and denote by $r$ and $\left(n_{1}, \ldots, n_{r}\right)$ its degree of non-holonomy and its growth vector, respectively. Finally, let $w_{1} \leq \ldots \leq w_{n}$ be the weights associated with the flag, defined by $w_{i}=s$ if $n_{s-1}<i \leq n_{s}$, setting $n_{0}=0$.

For any smooth vector field $f$, we denote its action, as a derivation on smooth functions, by $f: a \in \mathrm{C}^{\infty}(M) \mapsto$ $f a \in \mathrm{C}^{\infty}(M)$. For any smooth function $a$ and every vector field $f$ with $f \not \equiv 0$ near $q$, their (non-holonomic) order at $q$ is

$$
\begin{aligned}
& \operatorname{ord}_{q}(a)=\min \left\{s \in \mathbb{N}: \exists i_{1}, \ldots, i_{s} \in\{1, \ldots, m\} \text { s.t. }\left(f_{i_{1}} \ldots f_{i_{s}} a\right)(q) \neq 0\right\} \\
& \operatorname{ord}_{q}(f)=\max \left\{\sigma \in \mathbb{Z}: \operatorname{ord}_{q}(f a) \geq \sigma+\operatorname{ord}_{q}(a) \text { for any } a \in \mathrm{C}^{\infty}(M)\right\} .
\end{aligned}
$$

In particular, it can be proved that $\operatorname{ord}_{q}(a) \geq s$ if and only if $a\left(q^{\prime}\right)=\mathcal{O}\left(d_{\mathrm{SR}}\left(q^{\prime}, q\right)\right)^{s}$.

The following proposition clarifies the relationship between non-holonomic orders and the flag (2.5).

Proposition 2.3. Let (SR1) be satisfied, i.e. assume that $\left\{f_{1}, \ldots, f_{m}\right\}$ satisfies the Hörmander condition, and let $q \in M$ and $s \in \mathbb{N}$. Then, for any smooth vector field $f$ it holds that $\operatorname{ord}_{q^{\prime}} f=-s$ for any $q^{\prime}$ near $q$ if and only if $f\left(q^{\prime}\right) \in \Delta^{s}\left(q^{\prime}\right) \backslash \Delta^{s-1}\left(q^{\prime}\right)$ for any $q^{\prime}$ near $q$.

Remark 2.4. In the previous proposition, the fact that the assumptions hold in a neighborhood of $q$ is essential. Indeed, although it is true that $\operatorname{ord}_{q} f \geq-s$ implies $f(q) \in \Delta^{s}(q)$, when the growth vector is not constant around $q$ the contrary is false. To see this, it suffices to consider the sub-Riemannian control system on $\mathbb{R}^{2}$ with (privileged) coordinates $(x, y)$, defined by the vector fields $\partial_{x}$ and $x^{2} \partial_{y}$. Outside $\{x=0\}$, the non-holonomic degree of these vector fields is -1 , while on $\{x=0\}$ we need two brackets to generate the $y$ direction and hence the non-holonomic degree of $\partial_{y}$ is -3 . Then, the vector field $f(x, y)=\partial_{x}+x \partial_{y}$ is such that $f(0,0) \in \Delta^{1}(0,0)$ but $\operatorname{ord}_{(0,0)} f=-2$.

Definition 2.5. A system of privileged coordinates at $q$ for $\left\{f_{1}, \ldots, f_{m}\right\}$ is a system of local coordinates $z=$ $\left(z_{1}, \ldots, z_{n}\right)$ centered at $q$ and such that $\operatorname{ord}_{q}\left(z_{i}\right)=w_{i}, 1 \leq i \leq n$. 
The family of smooth vector fields $\left\{g_{1}, \ldots, g_{n}\right\}$ is an adapted basis at $q \in M$ if $\operatorname{span}\left\{g_{1}(q), \ldots, g_{n_{s}}(q)\right\}=\Delta^{s}(q)$ for any $1 \leq s \leq r$. (Equivalently: if $\operatorname{span}\left\{g_{1}(q), \ldots, g_{n}(q)\right\}=T_{q} M$ and $g_{i} \in \Delta^{w_{i}}(q)$ for any $1 \leq i \leq n$.) By continuity, if $\left\{g_{1}, \ldots, g_{n}\right\}$ is an adapted basis at $q$, it is a basis of $T_{q^{\prime}} M$ for any $q^{\prime}$ near $q$, which in general will not be adapted.

Through an adapted basis at $q$, it is always possible to define a system of privileged coordinates at $q$. Namely, for any permutation $\left\{i_{1}, \ldots, i_{n}\right\}$ of $\{1, \ldots, n\}$, the inverse $z=\left(z_{1}, \ldots, z_{n}\right)$ of the local diffeomorphism

$$
\phi:\left(z_{1}, \ldots, z_{n}\right) \mapsto \mathrm{e}^{z_{i_{n}} g_{i_{n}}} \circ \cdots \circ \mathrm{e}^{z_{i_{1}} g_{i_{1}}}(q),
$$

is a system of privileged coordinates at $q$, called canonical coordinates of the second kind. In particular, in this system $g_{i_{n}}$ is rectified, i.e., $z_{*} g_{i_{n}} \equiv \partial_{z_{i_{n}}}$, where $z_{*}$ is the push-forward operator on vector fields associated with the coordinates, defined as $z_{*} f=d z \circ f \circ z^{-1}$. We immediately obtain the following.

Proposition 2.6. Let (SR1) be satisfied, i.e. assume that $\left\{f_{1}, \ldots, f_{m}\right\}$ satisfies the Hörmander condition, and let $q \in M$ and $f$ be a smooth vector field such that $f(q) \neq 0$ and $f(q) \in \Delta^{s}(q) \backslash \Delta^{s-1}(q)$ for some $s \in \mathbb{N}$. Then, there exists a system of privileged coordinates $z=\left(z_{1}, \ldots, z_{n}\right)$ at $q$ rectifying $f$, i.e., such that $z_{*} f \equiv \partial_{k}$ for some $1 \leq k \leq n$.

Consider any system of privileged coordinates $z=\left(z_{1}, \ldots, z_{n}\right)$. We now show that it allows to compute the order of functions or vector fields in a purely algebraic way. Given a multiindex $\alpha=\left(\alpha_{1}, \ldots, \alpha_{n}\right)$ we define the weighted degree of the monomial $z^{\alpha}=z_{1}^{\alpha_{1}} \cdots z_{n}^{\alpha_{n}}$ as $w(\alpha)=w_{1} \alpha_{1}+\cdots+w_{n} \alpha_{n}$ and the weighted degree of the monomial vector field $z^{\alpha} \partial_{z_{j}}$ as $w(\alpha)-w_{j}$. Then given $a \in \mathrm{C}^{\infty}(M)$ and a smooth vector field $f$ with Taylor expansions

$$
a(z) \sim \sum_{\alpha} a_{\alpha} z^{\alpha} \quad \text { and } \quad f(z) \sim \sum_{\alpha, j} f_{\alpha, j} z^{\alpha} \partial_{z_{j}}
$$

their orders at $q$ can be computed as

$$
\operatorname{ord}_{q}(a)=\min \left\{w(\alpha): a_{\alpha} \neq 0\right\} \quad \text { and } \quad \operatorname{ord}_{q}(f)=\min \left\{w(\alpha)-w_{j}: f_{\alpha, j} \neq 0\right\} .
$$

A function or a vector field is said to be homogeneous if all the nonzero terms of its Taylor expansion have the same weighted degree.

We recall that, for any $a, b \in \mathrm{C}^{\infty}(M)$ and any smooth vector fields $f, g$, the order satisfies the following properties

$$
\begin{array}{cl}
\operatorname{ord}_{q}(a+b)=\min \left\{\operatorname{ord}_{q}(a), \operatorname{ord}_{q}(b)\right\}, & \operatorname{ord}_{q}(a b)=\operatorname{ord}_{q}(a)+\operatorname{ord}_{q}(b), \\
\operatorname{ord}_{q}(f+g)=\min \left\{\operatorname{ord}_{q}(f), \operatorname{ord}_{q}(g)\right\}, & \operatorname{ord}_{q}([f, g]) \geq \operatorname{ord}_{q}(f)+\operatorname{ord}_{q}(g) .
\end{array}
$$

Consider the control vector fields $f_{i}, 1 \leq i \leq m$. By the definition of order, it follows that $\operatorname{ord}_{q}\left(f_{i}\right) \geq-1$. Then we can express $f_{i}$ in coordinates as

$$
z_{*} f_{i}=\sum_{j=1}^{n}\left(h_{i j}+r_{i j}\right) \partial_{z_{j}},
$$

where $h_{i j}$ are homogeneous polynomials of weighted degree $w_{j}-1$ and $r_{i j}$ are functions of order larger than or equal to $w_{j}$.

Definition 2.7. The nilpotent approximation at $q$ of $f_{i}, 1 \leq i \leq m$, associated with the privileged coordinates $z$ is the vector field with coordinate representation

$$
z_{*} \widehat{f_{i}}=\sum_{j=1}^{n} h_{i j} \partial_{z_{j}}
$$


The nilpotentized sub-Riemannian control system is then defined as

$$
\dot{q}=\sum_{j=1}^{m} u_{j}(t) \widehat{f}_{j}(q) .
$$

The family of vector fields $\left\{\widehat{f}_{1}, \ldots, \widehat{f}_{m}\right\}$ is bracket-generating and nilpotent of step $r$ (i.e., every iterated bracket $\left[f_{i_{1}},\left[\ldots,\left[f_{i_{k-1}}, f_{i_{k}}\right]\right]\right]$ of length larger than $r$ is zero).

The main property of the nilpotent approximation is the following (see for example [8], Prop. 7.29).

Proposition 2.8. Let (SR1) be satisfied, i.e. assume that $\left\{f_{1}, \ldots, f_{m}\right\}$ satisfies the Hörmander condition, and let $z=\left(z_{1}, \ldots, z_{n}\right)$ be a system of privileged coordinates at $q \in M$ for $\left\{f_{1}, \ldots, f_{m}\right\}$. For $T>0$ and $u \in$ $\mathrm{L}^{1}\left([0, T] ; \mathbb{R}^{m}\right)$, with $|u| \equiv 1$, let $\gamma(\cdot)$ and $\hat{\gamma}(\cdot)$ be the trajectories associated with $u$ in (SR) and (NSR), respectively, and such that $\gamma(0)=\hat{\gamma}(0)=q$. Then, there exist $C, T_{0}>0$, independent of $u$, such that, for any $t<T_{0}$, it holds

$$
\left|z_{i}(\gamma(t))-z_{i}(\hat{\gamma}(t))\right| \leq C t^{w_{i}+1}, \quad i=1, \ldots, n .
$$

We recall, finally, the celebrated Ball-Box Theorem, that gives a rough description of the shape of small sub-Riemannian balls.

Theorem 2.9 (Ball-Box theorem).

Let (SR1) be satisfied, i.e. assume that $\left\{f_{1}, \ldots, f_{m}\right\}$ satisfies the Hörmander condition, and let $z=\left(z_{1}, \ldots, z_{n}\right)$ be a system of privileged coordinates at $q \in M$ for $\left\{f_{1}, \ldots, f_{m}\right\}$. Then there exist $C, \varepsilon_{0}>0$ such that for any $\varepsilon<\varepsilon_{0}$, it holds

$$
\operatorname{Box}\left(\frac{1}{C} \varepsilon\right) \subset B_{\mathrm{SR}}(q, \varepsilon) \subset \operatorname{Box}(C \varepsilon) .
$$

Here, $B_{\mathrm{SR}}(q, \varepsilon)$ is identified with its coordinate representation $z\left(B_{\mathrm{SR}}(q, \varepsilon)\right)$ and, for any $\eta>0$, we let

$$
\operatorname{Box}(\eta)=\left\{z \in \mathbb{R}^{n}:\left|z_{i}\right| \leq \eta^{w_{i}}\right\}
$$

The constants $C$ and $\varepsilon_{0}$ in the above theorem depend on $q$ and are not uniform, in general. However, this happens only when $q$ is a singular point for the sub-Riemannian structure, i.e., if the growth vector is not constant near $q$. We now state an uniform version of the Ball-Box theorem along integral curves of vector fields, which we will need in Section 4.2. This is a particularization of a much more general result contained in [26].

Proposition 2.10. Let (SR1) be satisfied, i.e. assume that $\left\{f_{1}, \ldots, f_{m}\right\}$ satisfies the Hörmander condition, and let $z=\left(z_{1}, \ldots, z_{n}\right)$ be a system of privileged coordinates at $q \in M$ for $\left\{f_{1}, \ldots, f_{m}\right\}$. Let $f$ be a smooth vector field such that $q$ be regular on its integral curve. Namely, there exists $t_{0}$ such that $\operatorname{dim} \Delta^{s}\left(\mathrm{e}^{t}(q)\right), s \in \mathbb{N}$, is constant for $t<t_{0}$. Then, there exist $C, \varepsilon_{0}>0$ such that for any $\varepsilon<\varepsilon_{0}$ and $t<t_{0}$, it holds

$$
z\left(\mathrm{e}^{t f_{0}}(q)\right)+\operatorname{Box}\left(\frac{1}{C} \varepsilon\right) \subset B_{\mathrm{SR}}\left(\mathrm{e}^{t f_{0}}(q), \varepsilon\right) \subset z\left(\mathrm{e}^{t f_{0}}(q)\right)+\operatorname{Box}(C \varepsilon) .
$$

As a corollary of the Ball-Box theorem, we get the following result on the regularity of the distance.

Corollary 2.11. Let (SR1) be satisfied, i.e. assume that $\left\{f_{1}, \ldots, f_{m}\right\}$ satisfies the Hörmander condition, and let $z=\left(z_{1}, \ldots, z_{n}\right)$ be a system of privileged coordinates at $q \in M$ for $\left\{f_{1}, \ldots, f_{m}\right\}$. Then there exists $C, \varepsilon>0$ such that

$$
\frac{1}{C}\left|z\left(q^{\prime}\right)\right| \leq d_{\mathrm{SR}}\left(q, q^{\prime}\right) \leq C\left|z\left(q^{\prime}\right)\right|^{1 / r}, \quad q^{\prime} \in B_{\mathrm{SR}}(q, \varepsilon) .
$$




\section{Time-Dependent systems}

\subsection{Time-dependent control systems}

Consider the following time-dependent non-holonomic control system

$$
\dot{q}=\sum_{i=1}^{m} u_{i} f_{i}^{t}(q), \quad q \in M, \quad u=\left(u_{1}, \ldots, u_{m}\right) \in \mathbb{R}^{m}, \quad t \in I,
$$

where $I=[0, b)$ for some $b \leq+\infty$ and $\left\{f_{1}^{t}, \ldots, f_{m}^{t}\right\}$ is a family of non-autonomous smooth vector fields depending smoothly on time. We let $f_{u}^{t}=\sum_{i=1}^{m} u_{i} f_{i}^{t}$. Moreover, we will need the following assumptions, already stated in the introduction, to be satisfied.

(T1) The map $t \mapsto f_{i}^{t}$ is smooth for $1 \leq i \leq m$ and $t \in I$.

(T2) The family of smooth vector fields $\left\{f_{1}^{t}, \ldots, f_{m}^{t}\right\}_{t \in I}$ satisfies the strong Hörmander condition, i.e., $\left\{f_{1}^{t_{0}}, \ldots, f_{m}^{t_{0}}\right\}$ satisfies the Hörmander condition for any $t_{0} \in I$.

As we will see later on in Section 4, when considering families of time-dependent vector fields of the form $f_{i}^{t}=\left(\mathrm{e}^{-t f_{0}}\right)_{*} f_{i}$ assumption (T2) will follow from the strong Hörmander condition for the affine control system with drift $f_{0}$ and control vector fields $\left\{f_{1}, \ldots, f_{m}\right\}$ (i.e., assumption (D1)).

In analogy with the autonomous case, we define (TD)-admissible curves as absolutely continuous curves $\gamma:[0, T] \subset I \rightarrow M$ such that $\dot{\gamma}(t)=f_{u(t)}^{t}(\gamma(t))$ for a.e. $t \in[0, T]$ and for some control $u \in \mathrm{L}^{1}\left([0, T], \mathbb{R}^{m}\right)$. Observe, however, that contrary to what happens in the sub-Riemannian case, the (TD)-admissibility property is not invariant under time reparametrization, e.g., a time reversal. Thus, we define the cost (and not the length) of $\gamma$ to be

$$
c(\gamma)=\min \|u\|_{\mathrm{L}^{1}\left([0, T], \mathbb{R}^{m}\right)},
$$

where the minimum is taken over all controls $u$ such that $\gamma$ is associated with $u$ and is attained due to convexity. The value function induced by the time-dependent system is then defined as

$$
\rho\left(q, q^{\prime}\right)=\inf \left\{c(\gamma): \gamma \text { is }(\mathrm{TD}) \text {-admissible and } \gamma: q \rightsquigarrow q^{\prime}\right\} \text {. }
$$

Clearly, the value function is non-negative. It is not a metric since, in general, it fails both to be symmetric and to satisfy the triangular inequality. Moreover, as the following example shows, $\rho$ could be degenerate. Namely, it could happen that $q \neq q^{\prime}$ but $\rho\left(q, q^{\prime}\right)=0$.

Example 3.1. Let $M=\mathbb{R}$, with coordinate $x$ and consider the vector field $f^{t}=(1-t)^{-2} \partial_{x}$ defined on $[0,1)$. For any $x_{0} \in \mathbb{R}, x_{0} \neq 0$, and for any sequence $t_{n} \uparrow 1$, let $u_{n} \in \mathrm{L}^{1}\left(\left[0, t_{n}\right]\right)$ be defined as $u_{n} \equiv\left(1-t_{n}\right) x_{0}$. By definition, each $u_{n}$ steers the system from 0 to $x_{0}$. Hence,

$$
\rho_{1}\left(0, x_{0}\right) \leq \inf _{n \in \mathbb{N}}\left\|u_{n}\right\|_{\mathrm{L}^{1}\left(\left[0, t_{n}\right]\right)}=\inf _{n \in \mathbb{N}} \int_{0}^{t_{n}}\left(1-t_{n}\right) x_{0} \mathrm{~d} t=x_{0} \inf _{n \in \mathbb{N}} t_{n}\left(1-t_{n}\right)=0 .
$$

This proves that, for any $x_{0} \in \mathbb{R}, \rho_{1}\left(0, x_{0}\right)=0$.

For $T>0, q \in M$ and $\varepsilon>0$, we denote the reachable set from $q$ with cost less than $\varepsilon$ by

$$
\mathcal{R}(q, \varepsilon)=\left\{q^{\prime} \in M: \rho\left(q, q^{\prime}\right)<\varepsilon\right\}
$$

We will also consider the reachable set from $q$ in time less than $T>0$ and cost less than $\varepsilon$, and denote it by $\mathcal{R}_{T}(q, \varepsilon)$. Clearly $\mathcal{R}_{T}(q, \varepsilon) \subset \mathcal{R}(q, \varepsilon)$.

In general, the existence of minimizers for the optimal control problem associated with (TD) is not guaranteed. We conclude this section with an example of this fact. 
Example 3.2. Let $M=\mathbb{R}$, with coordinate $x$, and consider the vector field $f^{t}=\mathrm{e}^{-t} \partial_{x}$ for $t \in[0,1)$. Fix $x_{0} \in \mathbb{R}, x_{0} \neq 0$. Observe that, for any $T>0$ and any control $u \in \mathrm{L}^{1}([0, T])$ steering the system from 0 to $x_{0}$, it holds

$$
\left|x_{0}\right|=\left|\int_{0}^{T} u(t) \mathrm{e}^{-t} \mathrm{~d} t\right| \leq \int_{0}^{T}|u(t)| \mathrm{e}^{-t} \mathrm{~d} t<\|u\|_{\mathrm{L}^{1}([0, T])} .
$$

This implies $\rho\left(0, x_{0}\right) \geq\left|x_{0}\right|$. Let now $u_{n} \in \mathrm{L}^{1}([0,1 / n])$ be defined as $u_{n}(t)=x_{0} n \mathrm{e}^{t}$. Clearly $u_{n}$ steers the system from 0 to $x_{0}$. Moreover,

$$
\rho\left(0, x_{0}\right) \leq \inf _{n \in \mathbb{N}}\left\|u_{n}\right\|_{\mathrm{L}^{1}([0,1 / n])}=\left|x_{0}\right| \inf _{n \in \mathbb{N}} \frac{\mathrm{e}^{\frac{1}{n}}-1}{\frac{1}{n}}=\left|x_{0}\right| .
$$

This proves that $\rho\left(0, x_{0}\right)=\left|x_{0}\right|$. Hence, the non-existence of minimizers follows from (3.1).

\subsection{Finiteness and continuity of the value function}

In this section, we extend the Chow-Rashevsky Theorem to time-dependent non-holonomic systems under the strong Hörmander condition. Namely, we will prove the following.

Theorem 3.3. Assume that $\left\{f_{1}^{t}, \ldots, f_{m}^{t}\right\}_{t \in I}$ satisfies (T1) and (T2). Then, the function $\rho: M \times M \rightarrow[0,+\infty)$ is continuous. Moreover, for any $t_{0} \in I$ and any $q, q^{\prime} \in M$, letting $d_{\mathrm{SR}}$ be the sub-Riemannian distance induced by $\left\{f_{1}^{t_{0}}, \ldots, f_{m}^{t_{0}}\right\}$, it holds $\rho\left(q, q^{\prime}\right) \leq d_{\mathrm{SR}}\left(q, q^{\prime}\right)$.

Let us introduce some notation. Following [5], the flows between times $s, t \in \mathbb{R}$ of an autonomous vector field $f$ and of a non-autonomous vector field $\tau \mapsto f^{\tau}$ will be denoted by, respectively,

$$
\mathrm{e}^{(t-s) f}: M \rightarrow M \quad \text { and } \quad \overrightarrow{\exp } \int_{s}^{t} f^{\tau} \mathrm{d} \tau: M \rightarrow M .
$$

Fix $q \in M$ and assume, for the moment, that $t_{0}=0$. Let $\ell \in \mathbb{N}$ and $\mathcal{F}=\left(i_{1}, \ldots, i_{\ell}\right) \in\{1, \ldots, m\}^{\ell}$. For any $\mathcal{T} \in I, \mathcal{T}>0$, we define the switching end-point map at time $\mathcal{T}$ and associated with $\mathcal{F}$ to be the function $E_{\mathcal{T}, \mathcal{F}}: \mathbb{R}^{\ell} \rightarrow M$ defined as

$$
\begin{aligned}
E_{\mathcal{T}, \mathcal{F}}(\xi) & =\overrightarrow{\exp } \int_{\frac{\ell-1}{\ell} \mathcal{T}}^{\mathcal{T}} \frac{\ell}{\mathcal{T}} \xi_{\ell} f_{i_{\ell}}^{\tau} \mathrm{d} \tau \circ \cdots \circ \overrightarrow{\exp } \int_{0}^{\frac{\mathcal{T}}{\ell}} \frac{\ell}{\mathcal{T}} \xi_{1} f_{i_{1}}^{\tau} \mathrm{d} \tau(q) \\
& =\overrightarrow{\exp } \int_{\frac{\ell-1}{\ell}}^{1} \ell \xi_{\ell} f_{i_{\ell}}^{\tau \mathcal{T}} \mathrm{d} \tau \circ \cdots \circ \overrightarrow{\exp } \int_{0}^{\frac{1}{\ell}} \ell \xi_{1} f_{i_{1}}^{\tau \mathcal{T}} \mathrm{d} \tau(q) .
\end{aligned}
$$

Here, we applied a standard change of variables formula for non-autonomous flows. Let then

$$
g_{\mathcal{T}, \mathcal{F}}^{\tau}= \begin{cases}\ell \xi_{1} f_{i_{1}}^{\tau \mathcal{T}} & \text { if } 0 \leq \tau<1 / \ell \\ \ell \xi_{2} f_{i_{2}}^{(\tau-1 / \ell) \mathcal{T}} & \text { if } 1 / \ell \leq \tau<2 / \ell \\ \vdots & \\ \ell \xi_{\ell} f_{i_{\ell}}^{(\tau-(\ell-1) / \ell) \mathcal{T}} & \text { if }(\ell-1) / \ell \leq \tau<1,\end{cases}
$$

so that we can write

$$
E_{\mathcal{T}, \mathcal{F}}(\xi)=\overrightarrow{\exp } \int_{0}^{1} g_{\mathcal{T}, \mathcal{F}}^{\tau}(\xi) \mathrm{d} \tau(q) .
$$

Clearly, $t \mapsto \overrightarrow{\exp } \int_{0}^{t} g_{\mathcal{T}, \mathcal{F}}^{\tau}(\xi) \mathrm{d} \tau(q), t \in[0,1]$, is a (TD)-admissible trajectory. Thus, $E_{\mathcal{T}, \mathcal{F}}(\xi), T>0$, is the end-point of a piecewise smooth (TD)-admissible curve of cost $\sum_{i}\left|\xi_{i}\right|$. 
We recall that, by the series expansion of $\overrightarrow{\exp }$ (see [5]), for any non-autonomous smooth vector field $f^{\tau}$, it holds $\overrightarrow{\exp } \int_{0}^{t} f^{\tau} \mathrm{d} \tau(q)=\mathrm{e}^{t f^{0}}(q)+\mathcal{O}\left(t^{2}\right)$. Thus, we can define

$$
E_{0, \mathcal{F}}(\xi)=\lim _{\mathcal{T} \downarrow 0} E_{\mathcal{T}, \mathcal{F}}(\xi)=\mathrm{e}^{\xi_{\ell} f_{\ell}^{0}} \circ \ldots \circ \mathrm{e}^{\xi_{1} f_{1}^{0}}(q)=\overrightarrow{\exp } \int_{0}^{1} g_{0, \mathcal{F}}^{\tau}(\xi) \mathrm{d} \tau(q),
$$

where, $g_{0, \mathcal{F}}^{\tau}(\xi)$ is defined in (3.3). Then $t \mapsto \overrightarrow{\exp } \int_{0}^{t} g_{0, \mathcal{F}}^{\tau}(\xi) \mathrm{d} \tau(q), t \in[0,1]$, is an (SR)-admissible curve for the sub-Riemannian structure defined by $\left\{f_{1}^{0}, \ldots, f_{m}^{0}\right\}$ and $E_{0, \mathcal{F}}(\xi)$ is the end-point of a piecewise smooth trajectory in (SR).

After [34], we say that a point $q^{\prime} \in M$ is (TD)-reachable from $q$ at time $t_{0}=0$, if there exist $\ell \in \mathbb{N}$, $\mathcal{F} \in\{1, \ldots, m\}^{\ell}, \mathcal{T}>0$ and $\xi \in \mathbb{R}^{\ell}$, such that $E_{\mathcal{T}, \mathcal{F}}(\xi)=q^{\prime}$. In this case it is clear that $\rho\left(q, q^{\prime}\right) \leq \sum_{i}\left|\xi_{i}\right|$. Moreover, if $\xi^{\prime} \mapsto E_{\mathcal{T}, \mathcal{F}}\left(\xi^{\prime}\right)$ has rank $n$ at $\xi$, the point $q^{\prime}$ is said to be (TD)-normally reachable at time $t_{0}=0$. Finally, the point $q^{\prime}$ is said to be (SR)-reachable or (SR)-normally reachable for the vector fields $\left\{f_{1}^{0}, \ldots, f_{m}^{0}\right\}$, if these properties holds for $\mathcal{T}=0$.

In the case $t_{0}>0$, taking $\mathcal{T}>0$ such that $\mathcal{T}+t_{0} \in I$ and changing the interval of integration in (3.2) from $[0, \mathcal{T}]$ to $\left[t_{0}, t_{0}+\mathcal{T}\right]$, it is clear how to define (TD)-reachable and (TD)-normally reachable points from $q$ at time $t_{0}$, and (SR)-reachable and (SR)-normally reachable points for the vector fields $\left\{f_{1}^{t_{0}}, \ldots, f_{m}^{t_{0}}\right\}$.

The proof of the following lemma is an adaptation of [34], Lemma 3.1.

Lemma 3.4. Let $q^{\prime} \in M$ be (SR)-normally reachable for the vector fields $\left\{f_{1}^{t_{0}}, \ldots, f_{m}^{t_{0}}\right\}$ from $q$, by some $\ell \in \mathbb{N}$, $\xi \in \mathbb{R}^{\ell}$ and $\mathcal{F} \in\{1, \ldots, m\}^{\ell}$. Then, there exist $\varepsilon_{0}, \mathcal{T}_{0}>0$ such that, for any $\varepsilon<\varepsilon_{0}$, the point $q^{\prime}$ is (TD)-normally reachable at time $t_{0}$, by the same $\ell$ and $\mathcal{F}$, and some $\xi^{\prime} \in \mathbb{R}^{\ell}$, with $\sum_{j}\left|\xi_{j}-\xi_{j}^{\prime}\right| \leq \varepsilon$, and any $\mathcal{T}<\mathcal{T}_{0}$.

Proof. Without loss of generality, we assume $t_{0}=0$.

Let $U \subset \mathbb{R}^{\ell}$ be a neighborhood of $\xi$ such that $E_{0, \mathcal{F}}$ has still rank $n$ when restricted to it. Then, there exists $B=\left\{x: \quad \sum_{j}\left|x_{j}-\xi_{j}\right| \leq \varepsilon\right\} \subset U$ such that $E_{0, \mathcal{F}}$ maps diffeomorphically a neighborhood of $B$ in $U$ onto a neighborhood of $q$. It follows, from standard properties of differential equations, that, for $\mathcal{T}>0$ sufficiently small, the map $E_{\mathcal{T}, \mathcal{F}}$ is well defined on $B$ and that $E_{\mathcal{T}, \mathcal{F}} \longrightarrow E_{0, \mathcal{F}}$ as $\mathcal{T} \downarrow 0$ in the $C^{1}$-topology over $B$. Thus, there exists $\mathcal{T}_{1}>0$ such that, for $\mathcal{T}<\mathcal{T}_{1}, E_{\mathcal{T}, \mathcal{F}}$ has rank $n$ at every point of $B$.

Since the map $E_{0, \mathcal{F}}$ is an homeomorphism from $B$ onto a neighborhood of $q$, and $E_{\mathcal{T}, \mathcal{F}} \longrightarrow E_{0, \mathcal{F}}$ uniformly as $\mathcal{T} \downarrow 0$, it follows that there exists a fixed neighborhood $V$ of $q$ and $\mathcal{T}_{2}>0$ such that $V \subset E_{\mathcal{T}, \mathcal{F}}(B)$, for any $\mathcal{T}<\mathcal{T}_{2}$. Then, for any $\mathcal{T}<\min \left\{\mathcal{T}_{1}, \mathcal{T}_{2}\right\}$, there exists $\xi^{\prime} \in B$ such that the point $q^{\prime}=E_{\mathcal{T}, \mathcal{F}}\left(\xi^{\prime}\right)$ is (TD)-normally reachable.

We will use the following consequence of Lemma 3.4. We remark that the result holds even if $\left\{f_{1}^{t}, \ldots, f_{m}^{t}\right\}_{t \in I}$ satisfies the Hörmander condition only at the time $t_{0} \in I$.

Lemma 3.5. Let $d_{\mathrm{SR}}$ be the sub-Riemannian distance induced by $\left\{f_{1}^{t_{0}}, \ldots, f_{m}^{t_{0}}\right\}$, then for any $t_{1} \in I$, such that $t_{1}-t_{0}>0$ is sufficiently small, and for any $q, q^{\prime} \in M$ it holds that

$$
\inf \left\{c(\gamma): \gamma:\left[t_{0}, t_{1}\right] \rightarrow M \text { is (TD)-admissible, } \gamma\left(t_{0}\right)=q \text { and } \gamma\left(t_{1}\right)=q^{\prime}\right\} \leq d_{\mathrm{SR}}\left(q, q^{\prime}\right) .
$$

In particular, $\rho\left(q, q^{\prime}\right) \leq d_{\mathrm{SR}}\left(q, q^{\prime}\right)$.

Proof. Fix $\varepsilon>0$. By Chow's theorem it is clear that $q^{\prime}$ is (SR)-reacheable from $q$. Moreover, since there exist (SR)-normally reachable points from $q^{\prime}$ arbitrarily close to $q^{\prime}$ (see e.g., [1], Lem. 3.21), follows that $q^{\prime}$ is always (SR)-normally reacheable from $q$ by $\xi$ such that $\sum_{j}\left|\xi_{j}\right| \leq d_{\mathrm{SR}}\left(q, q^{\prime}\right)+\varepsilon / 2$. Hence, by Lemma 3.4 , if $\varepsilon$ and $\eta>0$ are sufficiently small, we have that $q^{\prime}$ is (TD)-normally reachable from $q$ at time $t_{0}$ by $\xi^{\prime}$ such that $\sum_{j}\left|\xi_{j}^{\prime}\right| \leq d_{\mathrm{SR}}\left(q, q^{\prime}\right)+\varepsilon$ and $T<t_{1}$. This clearly implies that

$$
\inf \left\{c(\gamma): \gamma \text { is (TD)-admissible, } \gamma\left(t_{0}\right)=q \text { and } \gamma\left(t_{1}\right)=q^{\prime}\right\} \leq d_{\mathrm{SR}}\left(q, q^{\prime}\right)+\varepsilon .
$$

Finally, the lemma follows letting $\varepsilon \downarrow 0$.

We now prove the main theorem of the section. 
Proof of Theorem 3.3. By Lemma 3.5, we only need to prove the continuity of $\rho$. We will prove only the lower semicontinuity, since the upper semicontinuity follows by similar arguments.

We start by proving the lower semicontinuity of $\rho(q, \cdot)$ at $q^{\prime}$. Consider a sequence $q_{k} \rightarrow q^{\prime}$ and let $u_{k} \in \mathrm{L}^{1}\left(\left[0, T_{k}\right], \mathbb{R}^{m}\right)$ be controls such that each one steers system (TD) from $q$ to $q_{k}$ and $\liminf _{k} \rho^{f_{0}}\left(q, q_{k}\right)=$ $\lim \inf _{k}\left\|u_{k}\right\|_{L^{1}}$. Then, by Lemma 3.5, for any $\varepsilon>0$ there exists a sequence of $\tilde{T}_{k}>0$ and a sequence of controls $v_{k} \in \mathrm{L}^{1}\left(\left[T_{k}, \tilde{T}_{k}\right], \mathbb{R}^{m}\right)$ all steering system (TD) from $q_{k}$ to $q^{\prime}$ and such that $\left\|v_{k}\right\|_{\mathrm{L}^{1}\left(\left[T_{k}, \tilde{T}_{k}\right], \mathbb{R}^{m}\right)} \leq d_{\mathrm{SR}}\left(q_{k}, q^{\prime}\right)+\varepsilon$. Since $d_{\mathrm{SR}}\left(q_{k}, q^{\prime}\right) \rightarrow 0$, this implies that

$$
\rho\left(q, q^{\prime}\right) \leq \lim _{n \rightarrow \infty}\left(\left\|u_{k}\right\|_{L^{1}\left(\left[0, T_{k}\right], \mathbb{R}^{m}\right)}+\left\|v_{k}\right\|_{\left.L^{1}\left(\left[T_{k}, \tilde{T}_{k}\right], \mathbb{R}^{m}\right]\right)}\right)=\liminf _{n} \rho\left(q, q_{k}\right)+\varepsilon .
$$

Letting $\varepsilon \downarrow 0$ proves that $\rho(q, \cdot)$ is lower semicontinuous at $q^{\prime}$.

In order to prove the lower semicontinuity of $\rho\left(\cdot, q^{\prime}\right)$ at $q$, let us define

$$
\varphi_{\varepsilon}(p)=\inf \left\{c(\gamma): \gamma:[\varepsilon, T] \subset I \rightarrow M \text { is (TD)-admissible and } \gamma: p \rightsquigarrow q^{\prime}\right\} .
$$

We claim that for any $p \in M$ it holds that $\varphi_{\varepsilon}(p) \longrightarrow \rho\left(p, q^{\prime}\right)$ as $\varepsilon \downarrow 0$. Since it is clear that $\varphi_{\varepsilon}(\cdot) \geq \rho\left(\cdot, q^{\prime}\right)$, it suffices to prove that

$$
\lim _{\varepsilon \downarrow 0} \varphi_{\varepsilon}(p) \leq \rho\left(p, q^{\prime}\right) \quad \text { for any } p \in M .
$$

To this aim, fix $p \in M$ and $\eta>0$ and let $\gamma:[0, T] \rightarrow M$ be such that $\gamma: p \rightsquigarrow q^{\prime}$ and that $c(\gamma) \leq \rho\left(p, q^{\prime}\right)+\eta$. It is clear that $\gamma(2 \varepsilon) \rightarrow p$ as $\varepsilon \downarrow 0$, and hence that $\rho(p, \gamma(2 \varepsilon)) \rightarrow 0$ as $\varepsilon \downarrow 0$, by the first part of the proof. Thus, for any $\varepsilon>0$ sufficiently small, there exists a (TD)-admissible curve $\gamma_{\varepsilon}:[\varepsilon, 2 \varepsilon] \rightarrow M$ such that $\gamma_{\varepsilon}: p \rightsquigarrow \gamma(2 \varepsilon)$ and $c\left(\gamma_{\varepsilon}\right) \leq \rho(p, \gamma(2 \varepsilon))+\eta$. By concatenating $\gamma_{\varepsilon}$ with $\left.\gamma\right|_{[2 \varepsilon, T]}$, we get that

$$
\varphi_{\varepsilon}(p) \leq c\left(\gamma_{\varepsilon}\right)+c(\gamma) \leq \rho(p, \gamma(2 \varepsilon))+\rho\left(p, q^{\prime}\right)+2 \eta
$$

Letting $\varepsilon \downarrow 0$ and then $\eta \downarrow 0$, this proves (3.4) and thus the claim.

Let now $q_{k} \rightarrow q$ and fix $\eta>0$. By Lemma 3.5 this implies that $\rho\left(q_{k}, q\right) \rightarrow 0$ and that for any $\varepsilon>0$ sufficiently small, there exists a (TD)-admissible curve $\gamma_{\varepsilon}:[0, \varepsilon] \rightarrow M$ such that $\gamma_{\varepsilon}: q_{k} \rightsquigarrow q$ and $c\left(\gamma_{\varepsilon}\right) \leq \rho\left(q_{k}, q\right)+\eta$. Hence

$$
\rho\left(q_{k}, q^{\prime}\right) \leq c\left(\gamma_{\varepsilon}\right)+\varphi_{\varepsilon}(q) \leq \rho\left(q_{k}, q\right)+\varphi_{\varepsilon}(q)+\eta .
$$

By the previous claim, letting $\varepsilon, \eta \downarrow 0$, this implies that $\rho\left(q_{k}, q^{\prime}\right) \leq \rho\left(q_{k}, q\right)+\rho\left(q, q^{\prime}\right)$. Since $\rho\left(q_{k}, q\right) \rightarrow 0$, taking the liminf as $k \rightarrow+\infty$, this proves the lower semicontinuity of $\rho\left(\cdot, q^{\prime}\right)$ at $q$, completing the proof.

Remark 3.6. From the Proof of Theorem 3.3, it follows that hypothesis (T2) is not sharp. Indeed, the following would suffice to prove the theorem.

$\left(\mathrm{T}^{\prime}\right)$ The family of smooth vector fields $\left\{f_{1}^{t}, \ldots, f_{m}^{t}\right\}_{t \in I}$ satisfies the strong Hörmander condition at $t=0$ and in an open neighborhood of $\sup I$.

We will conclude this section by showing that, in our framework, it is essential to assume the Hörmander condition on both ends of $I$ and hence that assumption (T3.6') is minimal. Although outside the scope of the present work, we remark that stronger assumptions on the regularity of the vector fields, i.e., that they are uniformly Lipschitz, would allow to prove Theorem 3.3 assuming only that $\left\{f_{1}^{t}, \ldots, f_{m}^{t}\right\}_{t \in I}$ satisfies the Hörmander condition at one time $t_{0} \in I$.

The following example proves that if the family $\left\{f_{1}^{t}, \ldots, f_{m}^{t}\right\}_{t \in I}$ satisfies the Hörmander condition only near $t=0$, then the value function is in general not continuous. Through a slight modification, the same argument can also be used to prove that the same holds if the Hörmander condition is satisfied only at a neighborhood of $\sup I$ or of any $t_{0} \in I$. 


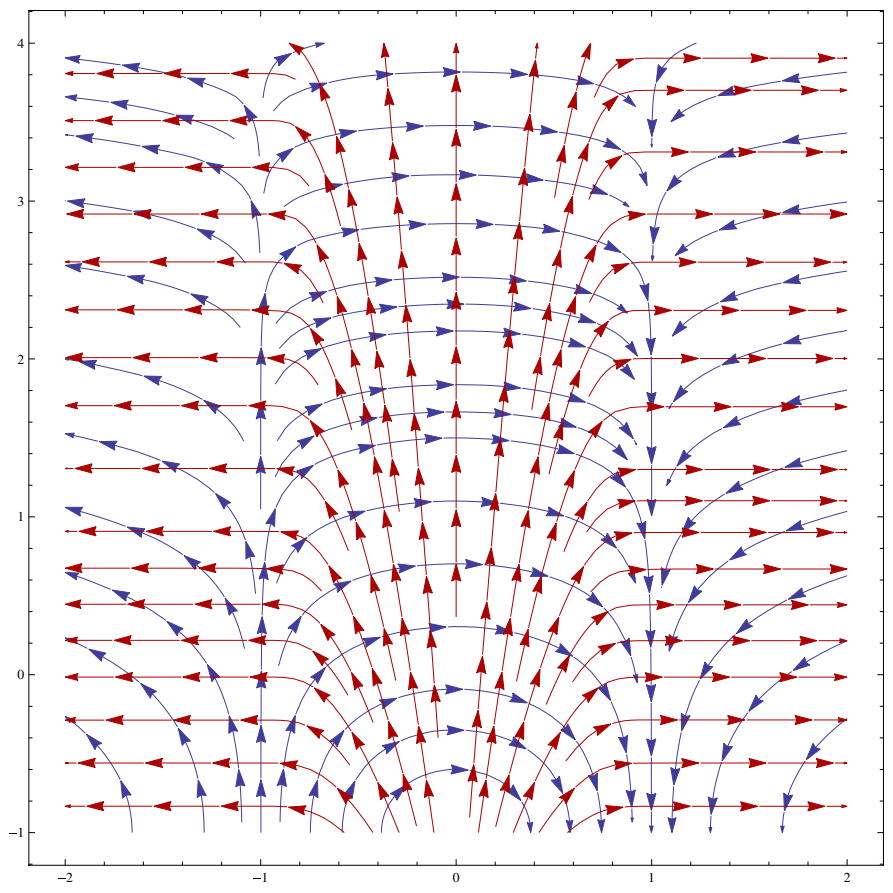

Figure 1. The two vector fields of Example 3.7 with $h(x)=c \mathrm{e}^{-\frac{1}{1-x^{2}}}$ for $x \in[-1,1]$.

Example 3.7. Let $M=(-2,2) \times(-1,+\infty)$, with coordinates $(x, y)$, and consider the vector fields

$$
f(x, y)=\frac{\left((y+1)\left(1-x^{2}\right),-x\right)}{\sqrt{(y+1)^{2}\left(1-x^{2}\right)^{2}+x^{2}}}, \quad g(x, y)=\frac{(x, h(x)(y+2))}{\sqrt{x^{2}+h(x)^{2}(y+2)^{2}}},
$$

where $h:[-2,2] \rightarrow \mathbb{R}$ is a smooth cutoff function such that $\operatorname{supp} h \subset[-1,1], h \geq 0$ and $h(0)=1$ (see Fig. 1). Fix $0<\varepsilon<1, C \geq 16$ and let $\phi, \psi:[0,1] \rightarrow \mathbb{R}$ be two smooth functions such that

$$
\phi(t)=\left\{\begin{array}{ll}
1 & \text { if } 0 \leq t \leq \varepsilon, \\
0 & \text { if } 2 \varepsilon \leq t \leq 1,
\end{array} \quad \psi(t)= \begin{cases}1 & \text { if } 0 \leq t \leq 2 \varepsilon, \\
C & \text { if } 3 \varepsilon \leq t \leq 1,\end{cases}\right.
$$

and such that $\phi$ is nonincreasing while $\psi$ is nondecreasing. Finally, consider the time-dependent system on $M$ specified by the vector fields $f^{t}(x, y)=\phi(t) f(x, y)$ and $g^{t}(x, y)=\psi(t) g(x, y), t \in[0,1]$. We will show that $\left\{f^{t}, g^{t}\right\}$ satisfies the Hörmander condition for $t \in[0, \varepsilon]$, but that the value function associated with the family $\left\{f^{t}, g^{t}\right\}_{t \in[0,1]}$ is not lower semicontinuous.

We start by showing that $f(p)$ and $g(p)$ are transversal for any $p=(x, y) \in M$, thus proving the Hörmander condition for $\left\{f^{t}, g^{t}\right\}, t \in[0, \varepsilon]$. If $x \in(-2,-1] \cup[1,2)$, then, by definition of $h, g(p)=(1,0)$ is clearly transversal to $f(p)$. On the other hand, if $x \in(-1,1) \backslash\{0\}$ and $g(p)$ is parallel to $f(p)$, a simple computation shows that $h(x)<0$, which is a contradiction. Finally, for $x=0$, it is clear that $g(p)=(0, y+2)$ and $f(p)=(y+1,0)$ are never parallel. We remark that this implies also that the value function $\rho_{\varepsilon}$, induced by controls defined on $[0, \varepsilon]$, is a distance equivalent to the Euclidean one. In particular, $\left|p_{1}-p_{2}\right| \leq 2 \rho_{\varepsilon}\left(p_{1}, p_{2}\right)$ for any $p_{1}, p_{2} \in M$.

Fix now $q^{\prime}=(1,0)$. The set of points from which $q^{\prime}$ is reachable using only $f$ is exactly $\mathcal{O}_{q^{\prime}}=\{(1, y): y>-1\}$. Let then $q_{0} \in(-1,0) \times\{0\}$ be such that $\rho_{\varepsilon}\left(q_{0},(-1,0)\right) \leq \frac{1}{4} \min _{p \in \mathcal{O}_{q^{\prime}}} \rho_{\varepsilon}\left(q_{0}, p\right)$. In order to show that $\rho_{1}\left(q_{0}, \cdot\right)$ is not lower semicontinuous at $q^{\prime}$, consider any sequence $\left\{q_{n}\right\}_{n \in \mathbb{N}} \subset(1 / 2,1) \times\{0\}$ such that $q_{n} \longrightarrow q^{\prime}$. By continuity of $\rho_{\varepsilon}$ and the fact that $-q_{n} \longrightarrow(-1,0)$, we can always assume that, up to subsequences, $\rho_{\varepsilon}\left(q_{0},-q_{n}\right) \leq$ $\frac{1}{2} \min _{p \in \mathcal{O}_{q^{\prime}}} \rho_{\varepsilon}\left(q_{0}, p\right)$. 
Since $g^{t} \equiv 0$ for $t \geq 2 \varepsilon$, if $u \in \mathrm{L}^{1}\left([0,1], \mathbb{R}^{2}\right)$ is a control steering the system from $q_{0}$ to $q^{\prime}$, the control $\left.u\right|_{[0,2 \varepsilon]}$ steers the system from $q_{0}$ to some $p \in \mathcal{O}_{q^{\prime}}$. Exploiting the fact that $\rho_{2 \varepsilon} \geq \rho_{\varepsilon}$ by monotonicity of $\psi$, this implies that

$$
\rho_{1}\left(q_{0}, q^{\prime}\right) \geq \min _{p \in \mathcal{O}_{q^{\prime}}} \rho_{\varepsilon}\left(q_{0}, p\right) \geq 2 \rho_{\varepsilon}\left(q_{0},-q_{n}\right) .
$$

Let now $u \in \mathrm{L}^{1}\left([0,1], \mathbb{R}^{m}\right)$ be the control constructed as follows. From time 0 to $\varepsilon,\left.u\right|_{[0, \varepsilon]}$ is the minimizer of $\rho_{\varepsilon}$ steering the system from $q_{0}$ to $-q_{n}$. Then, $\left.u\right|_{(\varepsilon, 3 \varepsilon)} \equiv 0$ and, after this, the control acts only on $f^{t}$ for time $t \in[3 \varepsilon, 1]$, steering the system from $-q_{n}$ to $q_{n}$. Hence,

$$
\left|q_{n}-\left(-q_{n}\right)\right|=\left|\int_{3 \varepsilon}^{1} u(t) f^{t}(x(t), y(t)) \mathrm{d} t\right|=C \int_{3 \varepsilon}^{1}|u(t)| \mathrm{d} t .
$$

Since $\left|q_{n}-\left(-q_{n}\right)\right|<2, C \geq 16 /\left|q_{0}-q^{\prime}\right| \geq 8 / \rho_{\varepsilon}\left(q_{0}, q^{\prime}\right)$, and by (3.6), it holds that

$$
\rho_{1}\left(q_{0}, q_{n}\right) \leq \int_{0}^{1}|u(t)| \mathrm{d} t=\rho_{\varepsilon}\left(q_{0},-q_{n}\right)+\frac{1}{C}\left|q_{n}-\left(-q_{n}\right)\right| \leq \frac{3}{4} \rho_{1}\left(q_{0}, q^{\prime}\right) .
$$

Taking the $\liminf$ as $n \rightarrow \infty$ shows that $\rho_{1}\left(q_{0}, \cdot\right)$ is not l.s.c. at $q^{\prime}$.

\subsection{Estimates on reachable sets}

In this section, we concentrate on a particular class of time-dependent systems. Namely, let $\left\{f_{1}, \ldots, f_{m}\right\}$ be a family of smooth vector fields, $f_{0}$ be a smooth vector field, and consider the time-dependent system

$$
\dot{q}=\sum_{i=1}^{m} u_{i} f_{i}^{t}, \quad f_{i}^{t}=\left(\mathrm{e}^{-t f_{0}}\right)_{*} f_{i}(q), \quad q \in M, \quad u=\left(u_{1}, \ldots, u_{m}\right) \in \mathbb{R}^{m} .
$$

Here, $\left(\mathrm{e}^{-t f_{0}}\right)_{*}$ is the push-forward operator associated with the flow of $f_{0}$. Throughout this section we will assume the following.

(T3) The family of smooth vector fields $\left\{f_{1}, \ldots, f_{m}\right\}$ satisfies the Hörmander condition.

Observe, in particular, that from (T3) it follows immediately that the family $\left\{\left(\mathrm{e}^{-t f_{0}}\right)_{*} f_{1}, \ldots,\left(\mathrm{e}^{-t f_{0}}\right)_{*} f_{m}\right\}$ satisfies (T2), i.e., the strong Hörmander condition for time-dependent systems. Moreover, (T1) is an immediate consequence of the definition of the $f_{i}^{t}$ 's.

As we will see in the next section, this class of systems arises naturally when dealing with control systems that are affine with respect to the control.

Before proceeding to estimate the shape of the reachable sets, we need to define a suitable approximation of system (3.7). Namely, fix a system of privileged coordinates (in the sub-Riemannian sense) at $q$ for $\left\{f_{1}, \ldots, f_{m}\right\}$. Assume that $f_{0}(q) \neq 0$, and let $s \in\{1, \ldots, r\}$ be such that $\operatorname{ord}_{q} f_{0}=-s$. In particular, by Remark 2.4, this implies that $f_{0}(q) \in \Delta^{s}(q) \backslash \Delta^{s-1}(q)$. In this case, there exist, in coordinates, an homogeneous vector field $f_{0}^{-s}$, of weighted degree $-s$, and a vector field $f_{0}^{>-s}$, of weighted degree $\geq-s+1$, such that $f_{0}^{-s} \not \equiv 0$ near $z(q)=0$ and

$$
z_{*} f_{0}=f_{0}^{-s}+f_{0}^{>-s} \text {. }
$$

For any smooth vector field $f$, let $\left(\operatorname{ad}^{1} f_{0}\right) f=\left[f_{0}, f\right]$ and $\left(\operatorname{ad}^{\ell} f_{0}\right) f=\left[f_{0},\left(\operatorname{ad}^{\ell-1} f_{0}\right) f\right]$, for any $\ell \in \mathbb{N}$. We recall (see for example [24]) that the following Taylor expansion holds

$$
\left(\mathrm{e}^{-t f_{0}}\right)_{*} f \sim \sum_{\ell=0}^{\infty} \frac{t^{\ell}}{\ell !}\left(\operatorname{ad}^{\ell} f_{0}\right) f .
$$

Since $\operatorname{ord}_{q}\left(f_{j}\right) \geq-1$, by (2.6) we have that $\operatorname{ord}_{q}\left(\left(\operatorname{ad}^{\ell} f_{0}\right) f_{j}\right) \geq-\ell s-1$. Then, by decomposition (3.8), for any $\ell \geq 0$, there exists, in coordinates, an homogeneous vector field $F_{j}^{\ell}$ of weighted degree $-\ell s$, and a remainder $r^{\ell}$ of order $\geq-\ell s-1$, such that

$$
z_{*}\left[\left(\operatorname{ad}^{\ell} f_{0}\right) f_{j}\right]=F_{j}^{\ell}+r^{\ell} .
$$


Definition 3.8. The homogeneous series approximation at $q$ of $f_{j}^{t}, 1 \leq j \leq m$, associated with the privileged coordinates $z$, is the vector field with coordinate representation

$$
\widehat{f}_{j}^{t}=\sum_{\ell=0}^{\varrho} \frac{t^{\ell}}{\ell !} F_{j}^{\ell},
$$

where $\varrho=\lfloor r-1 / s\rfloor$ and $r$ is the non-holonomic degree of $\left\{f_{1}, \ldots, f_{m}\right\}$ at $q$. The approximated time-dependent control system is then defined as

$$
\dot{q}=\sum_{j=1}^{m} u_{j}(t) \widehat{f}_{j}^{t}(q) .
$$

If a system, in some system of privileged coordinates, coincides with its homogeneous series approximation, we will say that it is series homogeneous.

The homogeneous series approximation encodes the idea that the time $t$ is of weight $s=-\operatorname{ord}_{q}\left(f_{0}\right)$. This is a consequence of the fact that, due to the expansion (3.9), $t$ allows to build brackets of $f_{0}$ with the $f_{j}$ s. In this sense, the homogeneous series approximation is a generalization of the nilpotent approximation.

We are now ready to state the main theorem of this section.

Theorem 3.9. Let (T3) be satisfied, i.e., assume that $\left\{f_{1}, \ldots, f_{m}\right\}$ satisfies the Hörmander condition, and let $z=\left(z_{1}, \ldots, z_{n}\right)$ be a system of privileged coordinates at $q \in M$ for $\left\{f_{1}, \ldots, f_{m}\right\}$. Then there exist $C, T, \varepsilon_{0}>0$ such that, for any $\varepsilon<\varepsilon_{0}$ and any $q^{\prime} \in \mathcal{R}_{T}(q, \varepsilon)$, setting $s \in \mathbb{N}$ to be such that $\operatorname{ord}_{q} f_{0}=-s$ it holds

$$
\begin{array}{ll}
\left|z_{i}\left(q^{\prime}\right)\right| \leq C\left(\varepsilon^{w_{i}}+\varepsilon T^{\frac{w_{i}}{s}}\right) & \text { if } w_{i} \leq s, \\
\left|z_{i}\left(q^{\prime}\right)\right| \leq C \varepsilon\left(\varepsilon+T^{\frac{1}{s}}\right)^{w_{i}-1} & \text { if } w_{i}>s .
\end{array}
$$

Moreover, if the system is series homogeneous, then it holds the stronger estimate

$$
\left|z_{i}\left(q^{\prime}\right)\right| \leq C \varepsilon^{w_{i}} \quad \text { if } w_{i} \leq s .
$$

To prove this theorem we need the following proposition, estimating the difference between (3.7) and (ATD).

Proposition 3.10. Let (T3) be satisfied, i.e., assume that $\left\{f_{1}, \ldots, f_{m}\right\}$ satisfies the Hörmander condition, and let $z=\left(z_{1}, \ldots, z_{n}\right)$ be a system of privileged coordinates at $q \in M$ for $\left\{f_{1}, \ldots, f_{m}\right\}$. For $T>0$ and $u \in \mathrm{L}^{1}\left([0, T] ; \mathbb{R}^{m}\right)$, let $\gamma(\cdot)$ and $\hat{\gamma}(\cdot)$ be the trajectories associated with $u$ in (3.7) and (ATD), respectively, and such that $\gamma(0)=\hat{\gamma}(0)=q$. Then there exist $C, \varepsilon_{0}, T_{0}>0$, independent of $u$, such that, if $t<T_{0}$ and $\int_{0}^{t}|u| \mathrm{d} s=\varepsilon<\varepsilon_{0}$, and setting $s \in \mathbb{N}$ to be such that $\operatorname{ord}_{q} f_{0}=-s$ it holds

$$
\left|z_{i}(\gamma(t))-z_{i}(\hat{\gamma}(t))\right| \leq C \varepsilon\left(\varepsilon+t^{\frac{1}{s}}\right)^{w_{i}}, \quad i=1, \ldots, n
$$

Remark 3.11. This proposition generalizes Proposition 2.8. In fact, in the sub-Riemannian case, since $f_{0} \equiv 0$, any curve $\gamma$ associated with $u \in \mathrm{L}^{1}\left([0, t], \mathbb{R}^{m}\right), t>0$, is associated also to $u_{\tau}(\cdot)=\frac{\tau}{t} u\left(\frac{\tau}{t} \cdot\right)$, for any $\tau>0$. Thus, since $\int_{0}^{\tau}\left|u_{\tau}\right| \mathrm{d} s=\int_{0}^{t}|u| \mathrm{d} s=\varepsilon,(3.15)$ reduces to

$$
\left|z_{i}(\gamma(t))-z_{i}(\hat{\gamma}(t))\right| \leq \inf _{\tau>0} C\left(\varepsilon^{w_{i}+1}+\tau \varepsilon^{r}\right)=C \varepsilon^{w_{i}+1} .
$$

Finally, assuming that $u$ satisfies the hypotheses of Proposition 2.8 , i.e., that $|u|=1$, we get $t=\varepsilon$. 
Proof. Let $z(\gamma(\cdot))=x(\cdot), z(\hat{\gamma}(\cdot))=y(\cdot)$, and $\|z\|=\sum_{\ell=1}^{n}\left|z_{\ell}\right|^{1 / w_{i}}$. We mimic the proof of Proposition 7.29 in [8]. The first step is to prove that there exists a constant $C>0$ such that $\|x(t)\|,\|y(t)\| \leq C \varepsilon$ for $t$ and $\varepsilon=\int_{0}^{t}|u| \mathrm{d} s$ small enough. We prove this for $\|x(t)\|$, the same argument works also for $\|y(t)\|$.

In $z$ coordinates, the equation of the control system (3.7) is,

$$
\dot{x}_{i}(t)=\sum_{j=1}^{m} u_{j}(t)\left(z_{i}\right)_{*} f_{j}^{t}(\gamma(t)), \quad i=1, \ldots, n .
$$

Due to the fact that $z_{*} f_{j}^{t}=z_{*} f_{j}+\mathcal{O}(t)$ uniformly in a neighborhood of $q$, that $\operatorname{ord}_{q}\left(z_{i}\right)=w_{i}$ and that $\operatorname{ord}_{q}\left(f_{j}\right) \geq-1$, we have that there exist $T_{0}$ and $C>0$ such that $\left|\left(z_{i}\right)_{*} f_{j}^{t}(q)\right| \leq \frac{C}{2}\left|\left(z_{i}\right)_{*} f_{j}(q)\right| \leq C\|x(t)\|^{w_{i}-1}$, for any $t<T_{0}$. Thus we get

$$
\left|\dot{x}_{i}(t)\right| \leq C \sum_{j=1}^{m}\left|u_{j}(t)\right|\left\|x_{i}(t)\right\|^{w_{j}-1} .
$$

As in the proof for the sub-Riemannian case, choosing $N$ sufficiently large, so that all $N / w_{i}$ are even integers, and setting $|\|z\||=\left(\sum_{\ell=1}^{n}\left|z_{\ell}\right|^{N / w_{i}}\right)^{\frac{1}{N}}$ we get a norm equivalent to $\|z\|$. Deriving with respect to time and using (3.16) we get $\frac{\mathrm{d}}{\mathrm{d} t}|||x(t)| \|\left|\leq C \sum_{j=1}^{n}\right| u_{j}(t) \mid$. Finally, by integration, equivalence of the norms, and the fact that $x(0)=z(q)=0$, we conclude that $\|x(t)\| \leq C \varepsilon$.

Now we move to proving (3.15). By construction of (ATD) and the Taylor expansion of $f_{j}^{t}$, for any $\ell \leq \varrho=$ $\left\lfloor{ }^{r-1} / s\right\rfloor$, there exist homogeneous polynomials $h_{j i}^{\ell}$ of order $w_{i}-\ell s-1$ and remainders $r_{j i}^{\ell}$ of order larger than or equal to $w_{i}-\ell s$, such that we can write

$$
\begin{aligned}
& \left(z_{i}\right)_{*} f_{j}^{t}=\sum_{\ell=0}^{\varrho} \frac{t^{\ell}}{\ell !}\left(h_{j i}^{\ell}+r_{j i}^{\ell}\right)+\mathcal{O}\left(t^{\varrho+1}\right), \\
& \left(z_{i}\right)_{*} \widehat{f}_{j}^{t}=\sum_{\ell=0}^{\varrho} \frac{t^{\ell}}{\ell !} h_{j i}^{\ell} .
\end{aligned}
$$

Here, the $\mathcal{O}$ is intended as $t \downarrow 0$ and is uniform in a compact neighborhood of the origin. Then,

$$
\begin{aligned}
\dot{x}_{i}(t)-\dot{y}_{i}(t) & =\sum_{j=1}^{m} u_{j}(t)\left(\sum_{\ell=0}^{\varrho} \frac{t^{\ell}}{\ell !}\left(h_{j i}^{\ell}(x)-h_{j i}^{\ell}(y)+r_{j i}^{\ell}(x)\right)+\mathcal{O}\left(t^{\varrho+1}\right)\right) \\
& =\sum_{j=1}^{m} u_{j}(t)\left(\sum_{\ell=0}^{\varrho} \frac{t^{\ell}}{\ell !}\left(\sum_{w_{k}<w_{i}-\ell s}\left(x_{k}(t)-y_{k}(t)\right) Q_{j i k}^{\ell}(x, y)+r_{j i}^{\ell}(x)\right)+\mathcal{O}\left(t^{\varrho+1}\right)\right),
\end{aligned}
$$

where $Q_{j i k}^{\ell}$ are homogeneous polynomial in $x$ and $y$, of order $w_{i}-w_{k}-\ell s-1$. We observe that, if $w_{i}-w_{k}-\ell s-1<$ 0 , then $Q_{j i k}^{\ell} \equiv 0$. Thus, for sufficiently small $\|x\|$ and $\|y\|$, we have

$$
\left|Q_{j i k}^{\ell}(x, y)\right| \leq C\left(\|x\|^{\left(w_{i}-w_{k}-\ell s-1\right)^{+}}+\|y\|^{\left(w_{i}-w_{k}-\ell s-1\right)^{+}}\right), \quad\left|r_{j i}^{\ell}(x)\right| \leq C\|x\|^{\left(w_{i}-\ell s\right)^{+}} .
$$

Here, we let $(\xi)^{+}=\max \{\xi, 0\}$, for any $\xi \in \mathbb{R}$. Using the inequalities of the first step, taking $t<T$ sufficiently small, and enlarging the constant $C$, we get

$$
\begin{aligned}
\left|\dot{x}_{i}(t)-\dot{y}_{i}(t)\right| & \leq C|u(t)|\left(\sum_{\ell=0}^{\varrho} \frac{t^{\ell}}{\ell !}\left(\sum_{w_{k}<w_{i}-\ell s}\left|x_{k}(t)-y_{k}(t)\right| \varepsilon^{w_{i}-w_{k}-\ell s-1}+\varepsilon^{\left(w_{i}-\ell s\right)^{+}}\right)+t^{\varrho+1}\right) \\
& \leq C|u(t)|\left(\sum_{\ell=0}^{\varrho} t^{\ell}\left(\sum_{w_{h}<w_{i}}\left|x_{h}(t)-y_{h}(t)\right| \varepsilon^{w_{i}-w_{h}-1}+\varepsilon^{\left(w_{i}-\ell s\right)^{+}}\right)+t^{\varrho+1}\right) .
\end{aligned}
$$

In the last inequality we applied the change of variable $w_{k} \mapsto w_{h}-\ell s$ in each of the sums. 
We can integrate the system by induction, since it is in triangular form. For $w_{i}=1$, since $\left(w_{i}-\ell s\right)^{+}=0$ for any $\ell \geq 1$, the inequality reduces to

$$
\left|\dot{x}_{i}(t)-\dot{y}_{i}(t)\right| \leq C|u(t)|\left(\sum_{\ell=0}^{\varrho} t^{\ell} \varepsilon^{\left(w_{i}-\ell s\right)^{+}}+t^{\varrho+1}\right) \leq C|u(t)|\left(\varepsilon^{w_{i}}+t\right) .
$$

Here we enlarged the constant $C$. Thus, integrating the previous inequality, we get $\left|x_{i}(t)-y_{i}(t)\right| \leq C \varepsilon\left(\varepsilon^{w_{i}}+t\right) \leq$ $C \varepsilon\left(\varepsilon+t^{\frac{1}{s}}\right)^{w_{i}}$.

Let, then, $w_{i}>1$ and assume that $\left|x_{h}(t)-y_{h}(t)\right| \leq C \varepsilon\left(\varepsilon+t^{\frac{1}{s}}\right)^{w_{h}}$ for any $w_{h}<w_{i}$. To complete the proof it suffices to show that $\left|\dot{x}_{i}(t)-\dot{y}_{i}(t)\right| \leq C|u(t)|\left(\varepsilon+t^{\frac{1}{s}}\right)^{w_{i}}$, since (3.15) will follow, as above, by integration. Thus, we have, enlarging again the constant $C$ and taking $t$ sufficiently small,

$$
\begin{aligned}
\left|\dot{x}_{i}(t)-\dot{y}_{i}(t)\right| & \leq C|u(t)|\left(\sum_{\ell=0}^{\varrho} t^{\ell}\left(\sum_{w_{h}<w_{i}}\left(\varepsilon+t^{\frac{1}{s}}\right)^{w_{h}} \varepsilon^{w_{i}-w_{h}}+\varepsilon^{\left(w_{i}-\ell s\right)^{+}}\right)+t^{\varrho+1}\right) \\
& \leq C|u(t)|\left(\sum_{\ell=0}^{\varrho} t^{\ell}\left(\sum_{w_{h}<w_{i}} t^{\frac{w_{h}}{s}} \varepsilon^{w_{i}-w_{h}}+\varepsilon^{\left(w_{i}-\ell s\right)^{+}}\right)+t^{\varrho+1}\right) .
\end{aligned}
$$

If $t \leq \varepsilon^{s}$, from (3.17) it is clear that $\left|\dot{x}_{i}(t)-\dot{y}_{i}(t)\right| \leq C|u(t)| \varepsilon^{w_{i}}$. Here we used the fact that $\varrho+1 \geq w_{i} / s$. On the other hand, if $\varepsilon<t^{1 / s}$, it holds

$$
\left|\dot{x}_{i}(t)-\dot{y}_{i}(t)\right| \leq C|u(t)|\left(\sum_{\ell=0}^{\varrho}\left(\sum_{w_{h}<w_{i}} t^{\frac{w_{h}}{s}+\ell+\frac{w_{i}-w_{h}}{s}}+t^{\ell+\frac{w_{i}-\ell s}{s}}\right)+t^{\varrho+1}\right) \leq C|u(t)| t^{\frac{w_{i}}{s}} .
$$

Putting together these two estimates, we get that $\left|\dot{x}_{i}(t)-\dot{y}_{i}(t)\right| \leq C|u(t)|\left(\varepsilon^{w_{i}}+t^{\frac{w_{i}}{s}}\right) \leq C|u(t)|\left(\varepsilon+t^{\frac{1}{s}}\right)^{w_{i}}$, completing the proof of the proposition.

Proof of Theorem 3.9. We start by claiming that (3.14) implies (3.12). In fact, if $\gamma: q \rightsquigarrow q^{\prime}$ is the trajectory associated in (3.7) to a control $u \in \mathrm{L}^{1}\left([0, T], \mathbb{R}^{m}\right)$, and $\hat{\gamma}$ is the trajectory associated with the same control in the homogeneous series approximation (ATD), with $\hat{\gamma}(0)=q$, it holds

$$
\left|z_{i}\left(q^{\prime}\right)\right| \leq\left|z_{i}(\hat{\gamma}(T))\right|+\left|z_{i}(\hat{\gamma}(T))-z_{i}(\gamma(T))\right| .
$$

Thus, by Proposition 3.10, the claim is proved.

Hence, from now on we assume our system to be in the form (ATD). Let us define, for $1 \leq j \leq n$ and $0 \leq \alpha \leq r$, the vector fields $\varphi_{j}^{\alpha}$ as

$$
\varphi_{j}^{\alpha}=\sum_{\ell=0}^{\alpha} \frac{t^{\ell}}{\ell !} F_{j}^{\ell}
$$

where $F_{j}^{\ell}$ are defined in (3.10). We do not explicitly denote the dependence on time, to lighten the notation. Observe that, if $\alpha=\varrho$, then, by (3.11), $\varphi_{j}^{\alpha}=\widehat{f}_{j}^{t}$.

We claim that, letting $x^{(\alpha)}(\cdot)$ be the trajectory associated with a control $u \in \mathrm{L}^{1}\left([0, T], \mathbb{R}^{m}\right)$ in system (TD) with $\left\{\varphi_{1}^{\alpha}, \ldots, \varphi_{m}^{\alpha}\right\}$ as vector fields, then, for some constant $C>0$ and any $i \in\{1, \ldots, n\}$ and $\alpha \geq 1$, it holds

$$
\left|x_{i}^{(\alpha)}(T)-x_{i}^{(\alpha-1)}(T)\right| \leq \begin{cases}0 & \text { if } w_{i} \leq \alpha s \\ C \varepsilon\left(\varepsilon+T^{\frac{1}{s}}\right)^{w_{i}-1} & \text { if } w_{i}>\alpha s .\end{cases}
$$

In fact, due to the homogeneity of the $F_{j}^{\ell}$, proceeding as in the proof of Proposition 3.10, we get that for $w_{i} \leq \alpha s$ it holds

$$
\left|\dot{x}_{i}^{(\alpha)}(t)-\dot{x}_{i}^{(\alpha-1)}(t)\right| \leq C|u(t)| \sum_{\ell=0}^{\alpha-1} t^{\ell} \sum_{w_{h}<w_{i}}\left|x_{h}^{(\alpha)}(t)-x_{h}^{(\alpha-1)}(t)\right| \varepsilon^{w_{i}-w_{h}-1} .
$$


By induction on $1 \leq w_{i} \leq \alpha s$, this proves the first part of the claim. On the other hand, if $w_{i}>\alpha s$, it holds

$$
\left|\dot{x}_{i}^{(\alpha)}(t)-\dot{x}_{i}^{(\alpha-1)}(t)\right| \leq C|u(t)|\left(\sum_{\ell=0}^{\alpha-1} t^{\ell} \sum_{w_{h}<w_{i}}\left|x_{h}^{(\alpha)}(t)-x_{h}^{(\alpha-1)}(t)\right| \varepsilon^{w_{i}-w_{h}-1}+t^{\alpha} \varepsilon^{w_{i}-\alpha s-1}\right) .
$$

Then, again by induction over $w_{i}$, we get that $\left|x_{i}^{(\alpha)}(T)-x_{i}^{(\alpha-1)}(T)\right| \leq C T^{\alpha} \varepsilon^{w_{i}-\alpha s}$. Finally, the claim follows considering the cases $T \leq \varepsilon^{s}$ and $T>\varepsilon^{s}$.

Due to the fact that $\varphi_{j}^{0}=\widehat{f}_{j}$, by Theorem 2.9 it holds $\left|x_{i}^{(0)}(T)\right| \leq C \varepsilon^{w_{i}}$. Thus, applying (3.18) and enlarging the constant $C$, we get

$$
\left|z_{i}\left(q^{\prime}\right)\right|=\left|x_{i}^{(r)}(T)\right| \leq \sum_{\ell=1}^{r}\left|x_{i}^{(\ell)}(T)-x_{i}^{(\ell-1)}(T)\right|+\left|x_{i}^{(0)}(T)\right| \leq \begin{cases}C \varepsilon^{w_{i}} & \text { if } w_{i} \leq s \\ C \varepsilon\left(\varepsilon+T^{\frac{1}{s}}\right)^{w_{i}-1} & \text { if } w_{i}>s\end{cases}
$$

This proves (3.13) and (3.14), completing the proof of the theorem.

We end this section by showing that the estimate (3.13) is sharp, at least in some directions. Indeed, for a system which is series homogeneous at $q$ in some privileged coordinates $z$, and satisfies the hypotheses of Theorem 3.9, it holds that $z_{*}\left(\left(\mathrm{ad}^{k} f_{0}\right) f_{j}\right)$ is an homogeneous vector field of weighted degree $-s k-1$. Thus, since $\varepsilon t^{k} \leq \varepsilon\left(\varepsilon+t^{\frac{1}{s}}\right)^{s k}$, the following proposition shows that (3.13) is sharp in this direction. The proof is an adaption of an argument from [16].

Proposition 3.12. Let (T3) be satisfied, i.e., assume that $\left\{f_{1}, \ldots, f_{m}\right\}$ satisfies the Hörmander condition. Let, moreover $q \in M, i \in\{1, \ldots, m\}$ and $k \geq 0$. Then, for any coordinate system $y$ at $q$, there exist $T, \varepsilon_{0}>0$ such that, for any $\varepsilon<\varepsilon_{0}$ and $t<T$ there exists a (TD)-admissible curve $\gamma:[0, t] \rightarrow M$, with $c(\gamma) \leq \varepsilon$, and such that

$$
y(\gamma(t))=\varepsilon t^{k} d y\left(\left(a d^{k} f_{0}\right) f_{j}(q)\right)+\mathcal{O}\left(\varepsilon t^{k+1}\right) \quad \text { as } \varepsilon t \rightarrow 0 .
$$

Proof. Let $t, \eta>0$ be fixed, and define $u \in \mathrm{L}^{1}\left([0, T], \mathbb{R}^{m}\right)$ as $u_{i}(\tau) \equiv \eta, u_{j}(\tau) \equiv 0$ for $j \neq i, \tau \in[0, t]$. Then, fix any $\Phi \in C^{k}([0,1])$ such that $\Phi^{(i)}(0)=\Phi^{(i)}(1)=0$, for $0 \leq i<k$. Thus, by integrating by parts and the fact that $\frac{\mathrm{d}}{\mathrm{d} t}\left(\mathrm{e}^{-t f_{0}}\right)_{*} g=\left(\mathrm{e}^{-t f_{0}}\right)_{*}\left(\operatorname{ad}\left(f_{0}\right) g\right)$, we get

$$
\int_{0}^{t} \Phi^{(k)}(\tau / t)\left(\mathrm{e}^{-\tau f_{0}}\right)_{*} f_{i}(q) \mathrm{d} \tau=t^{k} \int_{0}^{t} \Phi(\tau / t)\left(\mathrm{e}^{-\tau f_{0}}\right)_{*}\left(\left(\operatorname{ad}^{k} f_{0}\right) f_{i}\right)(q) \mathrm{d} \tau,
$$

for any $t$ and $q$. This implies that the flows generated by $\Phi^{(k)}(\tau / t)\left(\mathrm{e}^{-\tau f_{0}}\right)_{*} f_{i}$ and $t^{k} \Phi(\tau / t)\left(\mathrm{e}^{-\tau f_{0}}\right)_{*}\left(\left(\operatorname{ad}^{k} f_{0}\right) f_{i}\right)$ coincide. Using the series expansions of the chronological exponential and $\left(\mathrm{e}^{-t f_{0}}\right)_{*}$ (see [5], Sect. 2.4), it holds

$$
\begin{aligned}
\overrightarrow{\exp } \int_{0}^{t} \sum_{j=1}^{m} \Phi^{(k)}(\tau / t) u_{j}(\tau)\left(\mathrm{e}^{-\tau f_{0}}\right)_{*} f_{j} \mathrm{~d} \tau & =\overrightarrow{\exp } \int_{0}^{t} \eta \Phi^{(k)}(\tau / t)\left(\mathrm{e}^{-\tau f_{0}}\right)_{*} f_{i} \mathrm{~d} \tau \\
& =\overrightarrow{\exp } \int_{0}^{t} \eta t^{k} \Phi(\tau / t)\left(\mathrm{e}^{-\tau f_{0}}\right)_{*}\left(\left(\operatorname{ad}^{k} f_{0}\right) f_{i}\right) \mathrm{d} \tau \\
& =\overrightarrow{\exp } \int_{0}^{1} \eta t^{k+1} \Phi(s)\left(\mathrm{e}^{-t s f_{0}}\right)_{*}\left(\left(\operatorname{ad}^{k} f_{0}\right) f_{i}\right) \mathrm{d} s \\
& =\overrightarrow{\exp } \int_{0}^{1} \eta t^{k+1} \Phi(s)\left(\left(\operatorname{ad}^{k} f_{0}\right) f_{i}+\mathcal{O}(t)\right) \mathrm{d} s \\
& =\operatorname{Id}+\eta t^{k+1}\left(\operatorname{ad}^{k} f_{0}\right) f_{i}+\mathcal{O}\left(\eta t^{k+2}\right)
\end{aligned}
$$

Finally, considering any coordinate system and letting $\varepsilon=\eta t$, this completes the proof. 


\section{Control-AfFine Systems}

In this section we apply the results of Section 3 to control-affine systems. Let $\left\{f_{1}, \ldots, f_{m}\right\}$ be a family of vector fields, $f_{0}$ be a smooth vector field, called the drift, and consider the control-affine system

$$
\dot{q}=f_{0}(q)+\sum_{i=1}^{m} u_{i} f_{i}(q), \quad q \in M, \quad u=\left(u_{1}, \ldots, u_{m}\right) \in \mathbb{R}^{m} .
$$

Throughout this section we will assume the following.

(D1) The family of smooth vector fields $\left\{f_{0}, f_{1}, \ldots, f_{m}\right\}$ satisfies the strong Hörmander condition, i.e., the family $\left\{f_{1}, \ldots, f_{m}\right\}$ satisfies the Hörmander condition.

An absolutely continuous curve $\gamma:[0, T] \rightarrow M$ is (D)-admissible if $\dot{\gamma}(t)=f_{0}(\gamma(t))+f_{u(t)}(\gamma(t))$ for some control $u \in \mathrm{L}^{1}\left([0, T], \mathbb{R}^{m}\right)$. Its cost is defined as

$$
c_{f_{0}}(\gamma)=\min \|u\|_{L^{1}\left([0, T], \mathbb{R}^{m}\right)},
$$

where the minimum is taken over all controls $u$ such that $\gamma$ is associated with $u$. Then, two value functions we are interested in are

$$
\begin{gathered}
\rho_{T}^{f_{0}}\left(q, q^{\prime}\right)=\inf \left\{c(\gamma): \gamma:\left[0, T^{\prime}\right] \rightarrow M \text { is }(\mathrm{D}) \text {-admissible, } \gamma: q \rightsquigarrow q^{\prime}, T^{\prime} \leq T\right\}, \\
\rho^{f_{0}}\left(q, q^{\prime}\right)=\inf \left\{c(\gamma): \gamma(\mathrm{D}) \text {-admissible and } \gamma: q \rightsquigarrow q^{\prime}\right\} .
\end{gathered}
$$

It is clear that $\rho_{T}^{f_{0}}\left(q, q^{\prime}\right) \searrow \rho^{f_{0}}\left(q, q^{\prime}\right)$ as $T \rightarrow+\infty$, for any $q, q^{\prime} \in M$. Moreover, we observe that, $\rho_{T}^{f_{0}}\left(q, \mathrm{e}^{t f_{0}} q\right)=0$ for any $0 \leq t \leq T$. Finally, the reachable sets with respect to these value functions, from any $q \in M$ and for $\varepsilon, T>0$, are

$$
\mathcal{R}_{T}^{f_{0}}(q, \varepsilon)=\left\{q^{\prime} \in M: \rho_{T}^{f_{0}}\left(q, q^{\prime}\right)<\varepsilon\right\}, \quad \mathcal{R}^{f_{0}}(q, \varepsilon)=\left\{q^{\prime} \in M: \rho^{f_{0}}\left(q, q^{\prime}\right)<\varepsilon\right\} .
$$

\subsection{Connection with time-dependent systems}

Applying the variations formula (see [5]), system (D) can be written as a composition of a time-dependent system in the form (3.7) and of a translation along the drift. Namely, for any $u \in \mathrm{L}^{1}\left([0, T], \mathbb{R}^{m}\right)$, it holds

$$
\overrightarrow{\exp } \int_{0}^{T}\left(f_{0}+\sum_{i=1}^{m} u_{i}(t) f_{i}\right) \mathrm{d} t=\mathrm{e}^{T f_{0}} \circ \overrightarrow{\exp } \int_{0}^{T} \sum_{i=1}^{m} u_{i}(t)\left(\mathrm{e}^{-t f_{0}}\right)_{*} f_{i} \mathrm{~d} t .
$$

We call time-dependent system associated with (D) the following control system,

$$
\dot{q}=\sum_{i=1}^{m} u_{i}\left(\mathrm{e}^{-t f_{0}}\right)_{*} f_{i}(q), \quad q \in M, \quad u=\left(u_{1}, \ldots, u_{m}\right) \in \mathbb{R}^{m} .
$$

Observe that, since diffeomorphisms preserve linear independence, the strong Hörmander condition for (D), implies that $\left\{\left(\mathrm{e}^{-t f_{0}}\right)_{*} f_{1}, \ldots,\left(\mathrm{e}^{-t f_{0}}\right)_{*} f_{m}\right\}_{t \in[0,+\infty}$ satisfies (T3), i.e., the strong Hörmander condition for timedependent systems.

Exploiting these facts, we can prove the following.

Proposition 4.1. Let (D1) be satisfied, i.e., assume that $\left\{f_{0}, f_{1}, \ldots, f_{m}\right\}$ satisfies the strong Hörmander condition. Then, for any $T>0$, the functions $\rho_{T}^{f_{0}}, \rho^{f_{0}}: M \times M \rightarrow[0,+\infty)$ are continuous. Moreover, letting $d_{\mathrm{SR}}$ be the sub-Riemannian distance induced by $\left\{f_{1}, \ldots, f_{m}\right\}$, for any $q, q^{\prime} \in M$ it holds

$$
\rho_{T}^{f_{0}}\left(q, q^{\prime}\right) \leq \min _{0 \leq t \leq T} d_{\mathrm{SR}}\left(\mathrm{e}^{t f_{0}} q, q^{\prime}\right), \quad \rho^{f_{0}}\left(q, q^{\prime}\right) \leq \min _{t \geq 0} d_{\mathrm{SR}}\left(\mathrm{e}^{t f_{0}} q, q^{\prime}\right) .
$$


Proof. The continuity of the two functions, and the fact that $\rho_{T}^{f_{0}}\left(q, q^{\prime}\right), \rho^{f_{0}}\left(q, q^{\prime}\right) \leq d_{\mathrm{SR}}\left(q, q^{\prime}\right)$, for any $q, q^{\prime} \in M$, follows from the same arguments used in Theorem 3.3, adapting Lemmata 3.4 and 3.5 to the system (D). In particular, one has to consider $(\mathcal{T}, \mathcal{F}, \xi) \mapsto \mathrm{e}^{\mathcal{T} f_{0}} \circ E_{\mathcal{T}, \mathcal{F}}(\xi)$ instead of $(\mathcal{T}, \mathcal{F}, \xi) \mapsto E_{\mathcal{T}, \mathcal{F}}(\xi)$.

To prove the second part of the statement, we let, for any $t \in[0, T)$,

$$
\varphi_{t}(p)=\inf \left\{c(\gamma): \gamma:\left[t, T^{\prime}\right] \rightarrow M \text { is (D)-admissible, } \gamma: p \rightsquigarrow q^{\prime}, T^{\prime} \leq T\right\} .
$$

It is clear that, as above, it holds $\varphi_{t}(p) \leq d_{\mathrm{SR}}\left(p, q^{\prime}\right)$. Moreover, we observe that $\rho_{T}^{f_{0}}\left(q, \mathrm{e}^{t f_{0}} q\right)=0$ for any $0 \leq t<T$, and hence that for any such $t$ it holds

$$
\rho_{T}^{f_{0}}\left(q, q^{\prime}\right) \leq \varphi_{t}\left(\mathrm{e}^{t f_{0}} q\right) \leq d_{\mathrm{SR}}\left(\mathrm{e}^{t f_{0}} q, q^{\prime}\right) .
$$

Taking the minimum for $0 \leq t<T$, proves the inequality regarding $\rho_{T}^{f_{0}}$. To complete the proof it suffices to observe that $\rho^{f_{0}}\left(q, q^{\prime}\right) \leq \rho_{T}^{f_{0}}\left(q, q^{\prime}\right)$ for any $T>0$.

We point out that in system (D), as in time-dependent systems, the existence of minimizers is not assured. Moreover, this lack of minimizers is possible even if they exist for the associated time-dependent system, as the following example points out.

Example 4.2. Consider the following vector fields on $\mathbb{R}^{3}$, with coordinates $(x, y, z)$,

$$
f_{1}(x, y, z)=\partial_{x}, \quad f_{2}(x, y, z)=\partial_{y}+x \partial_{z} .
$$

Since $\left[f_{1}, f_{2}\right]=\partial_{z},\left\{f_{1}, f_{2}\right\}$ is a bracket-generating family of vector fields. The sub-Riemannian control system associated with $\left\{f_{1}, f_{2}\right\}$ on $\mathbb{R}^{3}$ corresponds to the Heisenberg group.

Let now $f_{0}=\partial_{z}$ be the drift. Since $\left[f_{1}, \partial_{z}\right]=\left[f_{2}, \partial_{z}\right]=0$ it holds that $\left(\mathrm{e}^{-t f_{0}}\right)_{*} f_{1}=f_{1}$ and $\left(\mathrm{e}^{-t f_{0}}\right)_{*} f_{2}=f_{2}$. Hence, the associated time-dependent system is actually not time-dependent. Thus, by (4.1), a curve $\gamma:[0, T] \rightarrow$ $\mathbb{R}^{3}$ is (SR)-admissible for $\left\{f_{1}, f_{2}\right\}$ if and only if $\tilde{\gamma}(\cdot)=\mathrm{e}^{\cdot f_{0}} \circ \gamma(\cdot)$ is (D)-admissible. As a consequence of this, for any $q \in \mathbb{R}^{3}$ and any $\varepsilon>0$,

$$
\mathcal{R}^{f_{0}}(q, \varepsilon)=\bigcup_{t \geq 0} \mathrm{e}^{t f_{0}} \circ B_{\mathrm{SR}}(q, \varepsilon) .
$$

As pointed out in Section 2, minimizers for the sub-Riemannian system exist between any pair of points in $B_{\mathrm{SR}}(q, \varepsilon)$, if $\varepsilon$ is sufficiently small. Let us show that, for any point in $\mathcal{R}^{f_{0}}(q, \varepsilon)$ with positive $z$ coordinate, we have an explicit minimizer, while for the others there exists no minimizer. Without loss of generality we can consider $q=0$. Then, since $\mathrm{e}^{t^{\prime} f_{0}}\left(x^{\prime}, y^{\prime}, z^{\prime}\right)=\left(x^{\prime}, y^{\prime}, z^{\prime}+t^{\prime}\right)$, every point $(x, y, z) \in \mathcal{R}^{f_{0}}(0, \varepsilon)$ with $z>0$, can be reached optimally considering the sub-Riemannian minimizing curve between the origin and $(x, y, 0)$ rescaled on time $z$.

If, instead, $z \leq 0$, we cannot construct any sub-Riemannian trajectory from 0 to $(x, y, z-t), t>0$, with cost $\leq d_{\mathrm{SR}}(0,(x, y, z))$. This is due to the fact that the minimizing trajectories in Heisenberg group are the lifts of arcs on the plane $(x, y)$, spanning area equal to the $z$ coordinates, and that $|z-t|=-z+t>|z|$. Since, by Proposition $4.1, \rho^{f_{0}}(0,(x, y, z)) \leq d_{\mathrm{SR}}(0,(x, y, z))$, this implies that there exists no minimizer for $\rho^{f_{0}}(q,(x, y, z))$.

\subsection{Estimates on reachable sets}

In this section we apply Theorem 3.9, in order to prove Theorem 1.1. Fixed a point $q \in M$, we will need the following assumptions, already stated in the introduction.

(D2) the point $q$ is regular for the integral curve of the drift, i.e., is such that $\operatorname{dim} \Delta^{s}\left(\mathrm{e}^{t f_{0}}(q)\right), s \in \mathbb{N}$, is constant for small $t$;

(D3) the point $q$ is regular w.r.t. the drift, in the sense that there exists $s \in \mathbb{N}$ such that $f_{0}\left(q^{\prime}\right) \in \Delta^{s}\left(q^{\prime}\right) \backslash \Delta^{s-1}\left(q^{\prime}\right)$, for any $q^{\prime}$ near $q$. 
By Proposition 2.3, (D3) is equivalent to $\operatorname{ord}_{q^{\prime}} f_{0}=-s$ for any $q^{\prime}$ near $q$. We remark also that, by (D3) and Proposition 2.6, it is always possible to find a system of privileged coordinates at $q$ satisfying the assumptions of Theorem 1.1.

Set $s \in \mathbb{N}$ to be such that $\operatorname{ord}_{q} f_{0}=-s$ and define the following sets, for parameters $\eta>0$ and $T>0$. We remark that $\operatorname{Box}(\eta)$ is defined as in $(2.7)$ and that $\left\{\partial_{z_{i}}\right\}_{i=1}^{n}$ is the canonical basis in $\mathbb{R}^{n}$.

$$
\begin{aligned}
& \Xi_{T}(\eta)=\bigcup_{0 \leq \xi \leq T}\left(\xi \partial_{z_{k}}+\operatorname{Box}(\eta)\right), \\
& \Pi_{T}(\eta)=\operatorname{Box}(\eta) \cup \bigcup_{0<\xi \leq T}\left\{z \in \mathbb{R}^{n}: 0 \leq z_{k}-\xi \leq \eta^{s},\left|z_{i}\right| \leq \eta^{w_{i}}+\eta \xi^{\frac{w_{i}}{s}} \text { for } w_{i} \leq s, i \neq k,\right. \\
& \quad \begin{array}{r}
\text { and } \left.\left|z_{i}\right| \leq \eta\left(\eta+\xi^{\frac{1}{s}}\right)^{w_{i}-1} \text { for } w_{i}>s\right\}, \\
\widehat{\Pi}_{T}(\eta)=\operatorname{Box}(\eta) \cup \bigcup_{0<\xi \leq T}\left\{z \in \mathbb{R}^{n}: 0 \leq z_{k}-\xi \leq \eta^{s},\left|z_{i}\right| \leq \eta^{w_{i}} \text { for } w_{i} \leq s, i \neq k,\right. \\
\text { and } \left.\left|z_{i}\right| \leq \eta\left(\eta+\xi^{\frac{1}{s}}\right)^{w_{i}-1} \text { for } w_{i}>s\right\} .
\end{array}
\end{aligned}
$$

As for Corollary 2.11 in the sub-Riemannian case, Theorem 1.1 is a direct consequence of some local estimates on the shape of the accessible sets, contained in the following. We cannot expect anything global, since in general the sets $\mathcal{R}^{f_{0}}(q, \varepsilon)$ are noncompact.

Theorem 4.3. Let (D1) be satisfied, i.e., assume that $\left\{f_{0}, f_{1}, \ldots, f_{m}\right\}$ satisfies the strong Hörmander condition, and let $q \in M$ be a point satisfying (D2) and (D3). Assume, moreover, that $z=\left(z_{1}, \ldots, z_{n}\right)$ is a system of privileged coordinates at $q$ for $\left\{f_{1}, \ldots, f_{m}\right\}$, such that $z_{*} f_{0}=\partial_{z_{k}}$, for some $1 \leq k \leq n$. Then, there exist $C, \varepsilon_{0}, T_{0}>0$ such that, setting $s \in \mathbb{N}$ to be such that $f_{0}(q) \in \Delta^{s}(q) \backslash \Delta^{s-1}(q)$,

$$
\Xi_{T}\left(\frac{1}{C} \varepsilon\right) \subset \mathcal{R}_{T}^{f_{0}}(q, \varepsilon) \subset \Pi_{T}(C \varepsilon), \quad \text { for } \varepsilon<\varepsilon_{0} \text { and } T<T_{0} .
$$

Here, with abuse of notation, we denoted by $\mathcal{R}_{T}^{f_{0}}(q, \varepsilon)$ the coordinate representation of the reachable set. In particular,

$$
\operatorname{Box}\left(\frac{1}{C} \varepsilon\right) \cap\left\{z_{k} \leq 0\right\} \subset \mathcal{R}_{T}^{f_{0}}(q, \varepsilon) \cap\left\{z_{k} \leq 0\right\} \subset \operatorname{Box}(C \varepsilon) \cap\left\{z_{k} \leq 0\right\} .
$$

Moreover, if the system is nilpotent, it holds

$$
\Xi_{T}\left(\frac{1}{C} \varepsilon\right) \subset \mathcal{R}_{T}^{f_{0}}(q, \varepsilon) \subset \widehat{\Pi}_{T}(C \varepsilon), \quad \text { for } \varepsilon<\varepsilon_{0} \text { and } T<T_{0} .
$$

Example 4.4. Consider the sub-Riemannian control system of Example 4.2, which is nilpotent and corresponds to the Heisenberg group, and endow it with the drift $f_{0}(x, y, z)=\partial_{z}+g(x, y, z)$, where $g(\cdot) \in \operatorname{span}\left\{f_{1}(\cdot), f_{2}(\cdot)\right\}$. Then, by Proposition 2.6, for any $g$ we can find a system of privileged coordinates $z=\left(z_{1}, z_{2}, z_{3}\right)$ at $(0,0,0)$ such that $z_{*} f_{0}=\partial_{z_{3}}$. Hence, since $(0,0,0)$ satisfies (D2) and (D3), by Theorem 4.3, for sufficiently small $\varepsilon$ and $T$, it holds

where (see Fig. 2),

$$
\Xi_{T}\left(C^{-1} \varepsilon\right) \subset \mathcal{R}_{T}^{f_{0}}((0,0,0), \varepsilon) \subset \widehat{\Pi}_{T}(C \varepsilon),
$$

$$
\begin{gathered}
\Xi_{T}\left(C^{-1} \varepsilon\right)=\left[-C^{-1} \varepsilon, C^{-1} \varepsilon\right] \times\left[-C^{-1} \varepsilon, C^{-1} \varepsilon\right] \times\left[-\left(T+C^{-1} \varepsilon^{2}\right), T+C^{-1} \varepsilon^{2}\right], \\
\widehat{\Pi}_{T}(C \varepsilon)=\operatorname{Box}(C \varepsilon) \cup \bigcup_{0 \leq \xi \leq T}\left(\left[-\left(C \varepsilon+C \varepsilon \xi^{\frac{1}{2}}\right), C \varepsilon+C \varepsilon \xi^{\frac{1}{2}}\right] \times\left[0, C \varepsilon^{2}\right]\right) .
\end{gathered}
$$

In particular, since the coordinates $(x, y, z)$ are priviliged, by (4.3) this is true although not at all sharp, when $g \equiv 0$.

In order to prove Theorem 4.3, we need the following lemma. 


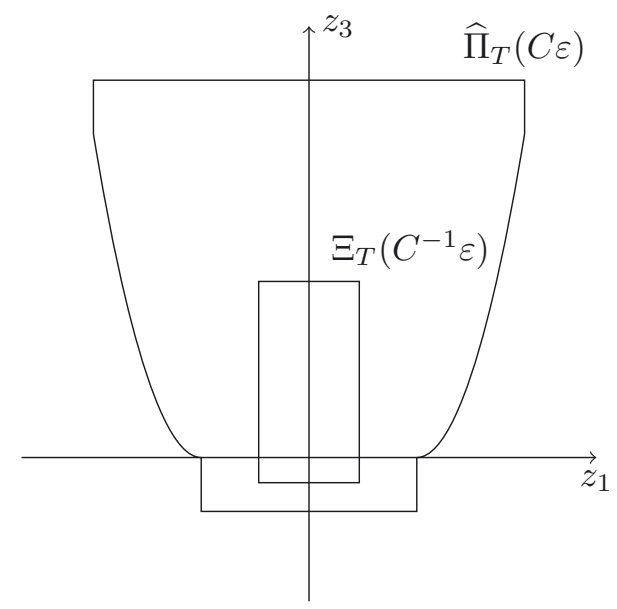

Figure 2. The section at $z_{2}=0$ of the boxes of Example 4.4 .

Lemma 4.5. Let (D1) be satisfied, i.e., assume that $\left\{f_{0}, f_{1}, \ldots, f_{m}\right\}$ satisfies the strong Hörmander condition. Let $z=\left(z_{1}, \ldots, z_{n}\right)$ be a system of privileged coordinates at $q \in M$ and set $s \in \mathbb{N}$ to be such that $\operatorname{ord}_{q} f_{0}=-s$. Then there exist $C, \varepsilon_{0}, T_{0}>0$ such that, for any $q^{\prime} \in \mathcal{R}_{T}^{f_{0}}\left(q, \varepsilon_{0}\right)$ for $\varepsilon<\varepsilon_{0}$ and $T<T_{0}$, and such that

(i) for any $t<\varepsilon_{0}$ it holds that $\operatorname{ord}_{q} f_{0}=-s$, where $q^{\prime}(t)=\mathrm{e}^{-t f_{0}}\left(q^{\prime}\right)$,

(ii) $d z_{k}\left(f_{0}\left(z\left(q^{\prime}\right)\right)\right) \neq 0$, for some $k$ with $w_{k}=-s$,

it holds that, if $u \in \mathrm{L}^{1}\left([0, T], \mathbb{R}^{m}\right)$ is a control steering the system (D) from $q$ to $q^{\prime}$, with $\|u\|_{1}=\varepsilon$, then

$$
T \leq C\left(\varepsilon^{s}+\max \left\{z_{k}\left(q^{\prime}\right), 0\right\}\right) .
$$

Proof. For any $\eta>0$, let $\gamma$ be the trajectory associated with $u \in \mathrm{L}^{1}\left([0, T], \mathbb{R}^{m}\right)$ in the system (D), and satisfying $\gamma: q \rightsquigarrow q^{\prime}$. Let $\tilde{\gamma}$ be the trajectory associated with $u$ and starting from $q$, in the time-dependent system (4.2). Thus $\gamma(t)=\mathrm{e}^{t f_{0}} \circ \tilde{\gamma}(t)$ and $\rho(q, \tilde{\gamma}(T)) \leq \varepsilon$.

Recall that, for any vector field $g$ and point $p \in M$, it holds that $z_{k}\left(\mathrm{e}^{T g}(p)\right)-z_{k}(p)=\int_{0}^{T} d z_{k}\left(g\left(\mathrm{e}^{t g}(p)\right)\right)$. Thus, by the mean value theorem, there exists $\tau \in[0, T]$ such that

$$
z_{k}\left(q^{\prime}\right)=z_{k}(\gamma(T))=T d z_{k}\left(f_{0}\left(\mathrm{e}^{\tau f_{0}}(\tilde{\gamma}(T))\right)\right)+z_{k}(\tilde{\gamma}(T)) .
$$

Since $\mathrm{e}^{\tau f_{0}}(\tilde{\gamma}(T))=\mathrm{e}^{-(T-\tau) f_{0}}\left(q^{\prime}\right)$, by hypothesis (ii) and the smoothness of $f_{0}$, there exist $T_{0}, C_{1}>0$, independent of $\gamma$, such that $d z_{k}\left(f_{0}\left(\mathrm{e}^{\tau f_{0}}(\tilde{\gamma}(T))\right)\right) \geq C_{1}$ for $T<T_{0}$. Hence, by Theorem 3.9 (since $\left.w_{k}=s\right)$, there exist $C_{2}, \bar{\varepsilon}>0$ such that, if $\varepsilon<\bar{\varepsilon}$ and $T<T_{0}$,

$$
T \leq \frac{\left|z_{k}(\tilde{\gamma}(T))\right|+\max \left\{z_{k}\left(q^{\prime}\right), 0\right\}}{C_{1}} \leq \frac{C_{2}\left(\varepsilon^{s}+T \varepsilon\right)+\max \left\{z_{k}\left(q^{\prime}\right), 0\right\}}{C_{1}} .
$$

Since the constants are independent of $\gamma$, taking $C=C_{2} / C_{1}$ and $\varepsilon_{0} \leq \min \left\{T_{0}, \bar{\varepsilon},(C-1) / C^{2}\right\}$ completes the proof.

Proof of Theorem 4.3. The first inclusion in (4.4) follows from Theorem 4.1, Proposition 2.10, and the fact that $z_{i}\left(\mathrm{e}^{t f_{0}}(q)\right)=0$, if $i \neq k$, and $z_{k}\left(\mathrm{e}^{t f_{0}}(q)\right)=t$. In fact, combining them, we have that, for any $\varepsilon<\varepsilon_{0}$ and any $T>0$,

$$
\Xi_{T}\left(\frac{1}{C} \varepsilon\right) \subset \bigcup_{0 \leq t \leq T} B_{\mathrm{SR}}\left(\mathrm{e}^{t f_{0}} q, \varepsilon\right) \subset \mathcal{R}_{T}^{f_{0}}(q, \varepsilon) .
$$


To prove the second inclusion, we let $q^{\prime} \in \mathcal{R}_{T}^{f_{0}}(q, \varepsilon)$. Fix any $\eta>0$ and consider a control $u \in \mathrm{L}^{1}\left([0, \tau], \mathbb{R}^{m}\right)$, $\tau \leq T$, such that its associated trajectory $\gamma$, in the system (D), satisfies $\gamma: q \rightsquigarrow q^{\prime}$ and $c_{f_{0}}(\gamma) \leq \varepsilon+\eta$. We distinguish two cases. First we assume that $z_{k}\left(q^{\prime}\right) \leq 0$. In this case, by Lemma 4.5 it follows there exists $C, \varepsilon_{0}, T_{0}>0$ such that if $\tau<T_{0}$ and $\varepsilon<\varepsilon_{0}$ then $\tau \leq C \varepsilon^{s}$. Moreover (4.1) implies that $\mathrm{e}^{-\tau f_{0}}\left(q^{\prime}\right) \in \mathcal{R}_{T}(q, \varepsilon)$. Then, enlarging the constant $C$, Theorem 3.9 yields

$$
\begin{aligned}
& \left|z_{i}\left(q^{\prime}\right)\right|=\left|z_{i}\left(\mathrm{e}^{-\tau f_{0}}\left(q^{\prime}\right)\right)\right| \leq C\left(\varepsilon^{w_{i}}+\varepsilon \tau^{\frac{w_{i}}{s}}\right) \leq C \varepsilon^{w_{i}}, \quad \text { if } w_{i} \leq s \text { and } i \neq k, \\
& \left|z_{k}\left(q^{\prime}\right)\right| \leq \tau+\left|z_{k}\left(\mathrm{e}^{-\tau f_{0}}\left(q^{\prime}\right)\right)\right| \leq \tau+C\left(\varepsilon+\tau^{\frac{1}{s}}\right)^{s} \leq C \varepsilon^{s}, \\
& \left|z_{i}\left(q^{\prime}\right)\right|=\left|z_{i}\left(\mathrm{e}^{-\tau f_{0}}\left(q^{\prime}\right)\right)\right| \leq C \varepsilon\left(\varepsilon+\tau^{\frac{1}{s}}\right)^{w_{i}-1} \leq C \varepsilon^{w_{i}}, \quad \text { if } w_{i}>s .
\end{aligned}
$$

Here, we used the fact that, for any $p \in M$, from $z_{*} f_{0}=\partial_{z_{k}}$, it holds $z_{i}(p)=z_{i}\left(\mathrm{e}^{-T f_{0}}(p)\right)$ and $\left|d z_{k}\left(f_{0}(p)\right)\right| \equiv 1$. Thus, if $T \leq T_{0}$, it holds $q^{\prime} \subset \operatorname{Box}(C \varepsilon) \subset \Pi(C \varepsilon)$.

On the other hand, if $z_{k}\left(q^{\prime}\right)>0$, Lemma 4.5 yields that $\tau \leq C\left(\varepsilon^{s}+z_{k}\left(q^{\prime}\right)\right)$. Then, applying again Theorem 3.9, we get

$$
\begin{gathered}
\left|z_{i}\left(q^{\prime}\right)\right|=\left|z_{i}\left(\mathrm{e}^{-\tau f_{0}}\left(q^{\prime}\right)\right)\right| \leq C\left(\varepsilon^{w_{i}}+\varepsilon \tau^{\frac{w_{i}}{s}}\right), \quad \text { if } w_{i} \leq s \text { and } i \neq k, \\
z_{k}\left(q^{\prime}\right) \leq \tau+\left|z_{k}\left(\mathrm{e}^{-\tau f_{0}}\left(q^{\prime}\right)\right)\right| \leq \tau+C\left(\varepsilon+\tau^{\frac{1}{s}}\right)^{s} \leq \tau+C \varepsilon^{s}, \\
\left|z_{i}\left(q^{\prime}\right)\right|=\left|z_{i}\left(\mathrm{e}^{-\tau f_{0}}\left(q^{\prime}\right)\right)\right| \leq C \varepsilon\left(\varepsilon+\tau^{\frac{1}{s}}\right)^{w_{i}-1}, \quad \text { if } w_{i}>s .
\end{gathered}
$$

Letting $\tau=\xi$, this proves that $q^{\prime} \subset \Pi(C \varepsilon)$, completing the proof of (4.4).

To prove (4.5) it suffices to use the same argument as above, applying the result on nilpotent systems in Theorem 3.9 .

Remark 4.6. Theorem 4.3 suggests that the behavior of system (D), when moving in the direction $-f_{0}$, is essentially sub-Riemannian. However, although this is true locally in time, it is false in general. For example, consider the Euclidean plane endowed with a rotational drift, i.e., such that $\left\{\mathrm{e}^{t f_{0}}(q)\right\}_{t \in(0,+\infty)}$ is diffeomorphic to $\mathcal{S}^{1}$ for any $q \neq 0$. Then, $\rho^{f_{0}}\left(q, \mathrm{e}^{-t f_{0}}(q)\right)=0$ for any $t>0$ and thus we can move in the direction $-f_{0}$ for free.

Remark 4.7. By the arguments used in the proof of Theorem 2.2 from [9], applied to our setting, it is possible to recover the first inclusion of Theorem 4.3. There, a different notion of approximation of control-affine systems is used. Namely, the authors do not consider the associated time-dependent system, preferring to define $\widehat{f}_{i}$ as a vector field such that $\operatorname{ord}_{q}\left(f_{i}-\widehat{f}_{i}\right) \geq 0$. In the literature this is sometimes called a first-order approximation. However, such notion does not take into account the role played by the time in control-affine systems, and thus it does not seem well suited to derive the estimates necessary to obtain the second inclusion. We thank the anonymous referee for bringing this fact to our attention.

Proof of Theorem 1.1. Since every norm on $\mathbb{R}^{n}$ is equivalent, $\operatorname{dist}\left(z\left(q^{\prime}\right),[0, T] \partial_{z_{k}}\right)$ is equivalent to

$$
a\left(q^{\prime}\right)=\sum_{\substack{1 \leq i \leq n \\ i \neq k}}\left|z_{i}\left(q^{\prime}\right)\right|+\min _{t \in[0, T]}\left|z_{k}\left(q^{\prime}\right)-t\right| .
$$

Thus, to complete the proof it suffices to prove that it holds $C^{-1} a\left(q^{\prime}\right) \leq \rho_{T}^{f_{0}}\left(q, q^{\prime}\right) \leq C a\left(q^{\prime}\right)^{1 / r}$.

By Theorem 3.9, $\Xi_{T}\left(C^{-1} \varepsilon\right) \subset \mathcal{R}_{T}^{f_{0}}(q, \varepsilon) \subset \Pi_{T}(C \varepsilon)$ for any $\varepsilon<\varepsilon_{0}$. The first inclusion is equivalent to the fact that, for every $\varepsilon<\varepsilon_{0}$ such that $C a\left(q^{\prime}\right) \leq \varepsilon^{r}$, one has $\rho_{T}^{f_{0}}\left(q, q^{\prime}\right) \leq \varepsilon$. From this follows that 
$\rho_{T}^{f_{0}}\left(q, q^{\prime}\right) \leq C^{1 / r} a\left(q^{\prime}\right)^{1 / r}$. The same reasoning applied to the other inclusion proves that

$$
\begin{aligned}
& \left|z_{i}\left(q^{\prime}\right)\right| \leq C\left(\rho_{T}^{f_{0}}\left(q, q^{\prime}\right)^{w_{i}}+\rho_{T}^{f_{0}}\left(q, q^{\prime}\right) T^{\frac{w_{i}}{s}}\right) \text { if } w_{i} \leq s, i \neq k, \min _{t \in[0, T]}\left|z_{k}\left(q^{\prime}\right)-t\right| \leq C \rho_{T}^{f_{0}}\left(q, q^{\prime}\right)^{s}, \\
& \left|z_{i}\left(q^{\prime}\right)\right| \leq C\left(\rho_{T}^{f_{0}}\left(q, q^{\prime}\right)^{w_{i}}+\rho_{T}^{f_{0}}\left(q, q^{\prime}\right) T^{\frac{w_{i}-1}{s}}\right) \text { if } w_{i}>s
\end{aligned}
$$

Clearly, this implies that $a\left(q^{\prime}\right) \leq C \rho_{T}^{f_{0}}\left(q, q^{\prime}\right)$, for some larger constant, completing the proof of the theorem.

\section{REFERENCES}

[1] A. Agrachev, D. Barilari and U. Boscain, Introduction to Riemannian and sub-Riemannian geometry (Lecture Notes). http:// people.sissa.it/agrachev/agrachev_files/notes.htm (2012).

[2] A. Agrachev, U. Boscain, J.-P. Gauthier and F. Rossi, The intrinsic hypoelliptic Laplacian and its heat kernel on unimodular Lie groups. J. Funct. Anal. 256 (2009) 2621-2655.

[3] A. Agrachev and P.W.Y. Lee, Continuity of optimal control costs and its application to weak KAM theory. Calc. Var. Partial Differ. Equ. 39 (2010) 213-232.

[4] A.A. Agrachev, U. Boscain, G. Charlot, R. Ghezzi and M. Sigalotti, Two-dimensional almost-Riemannian structures with tangency points. Ann. Inst. Henri Poincaré Anal. Non Linéaire 27 (2010) 793-807.

[5] A.A. Agrachev and Y.L. Sachkov, Control theory from the geometric viewpoint. Control Theory and Optimization II. Vol. 87 of Encycl. Math. Sci. Springer-Verlag, Berlin (2004).

[6] A. Agrachev, U. Boscain and M. Sigalotti, A Gauss-Bonnet-like formula on two-dimensional almost-Riemannian manifolds. Discrete Contin. Dyn. Syst. 20 (2008) 801-822.

[7] M. Barbero-Liñán and M. Sigalotti, High-order sufficient conditions for configuration tracking of affine connection control systems. Syst. Control Lett. 59 (2010) 491-503.

[8] A. Bellaïche, The tangent space in sub-Riemannian geometry. In Sub-Riemannian geometry. Vol. 144 of Progr. Math. Birkhäuser, Basel (1996) 1-78.

[9] R.M. Bianchini and G. Stefani, Graded approximations and controllability along a trajectory. SIAM J. Control Optim. 28 (1990) 903-924.

[10] R.M. Bianchini and G. Stefani. Time-optimal problem and time-optimal map. Rend. Sem. Mat. Univ. Politec. Torino 48 (1992) 401-429 (1990).

[11] U. Boscain and P. Mason, Time minimal trajectories for a spin $1 / 2$ particle in a magnetic field. J. Math. Phys. 47 (2006) 062101, 29.

[12] U. Boscain and G. Charlot, Resonance of minimizers for $n$-level quantum systems with an arbitrary cost. ESAIM: COCV 10 (2004) 593-614.

[13] U. Boscain, G. Charlot, J.-P. Gauthier, S. Guérin, and H.-R. Jauslin, Optimal control in laser-induced population transfer for two- and three-level quantum systems. J. Math. Phys. 43 (2002) 2107-2132.

[14] F. Bullo and A. D. Lewis, Geometric control of mechanical systems. Modeling, analysis, and design for simple mechanical control systems. Vol. 49 of Texts Appl. Math. Springer-Verlag, New York (2005).

[15] Y. Chitour, F. Jean and E. Trélat, Singular trajectories of control-affine systems. SIAM J. Control Optim. 47 (2008) $1078-1095$.

[16] J.-M. Coron, Control and nonlinearity. Vol. 136 of Math. Surv. Monogr. American Mathematical Society, Providence, RI (2007).

[17] D. D'Alessandro, Introduction to quantum control and dynamics. Chapman 8 Hall/CRC Appl. Math. Nonl. Sci. Series. Chapman \& Hall/CRC, Boca Raton, FL (2008).

[18] D. D'Alessandro and M. Dahleh, Optimal control of two-level quantum systems. IEEE Trans. Automat. Control 46 (2001) 866-876.

[19] L.D. Drager, J.M. Lee, E. Park and K. Richardson, Smooth distributions are finitely generated. Ann. Global Anal. Geom. 41 (2012) 357-369.

[20] G.B. Folland and E.M. Stein, Estimates for the $\bar{\partial}_{b}$ complex and analysis on the Heisenberg group. Comm. Pure Appl. Math. 27 (1974) 429-522.

[21] H. Frankowska, Value function in optimal control, in Mathematical control theory, Part 1, 2 (Trieste, 2001), vol. 8 of ICTP Lect. Notes. Abdus Salam Int. Cent. Theoret. Phys., Trieste (2002) 516-653 (electronic).

[22] J.-P. Gauthier and V. Zakalyukin, On the codimension one motion planning problem. J. Dyn. Control Syst. 11 (2005) 73-89.

[23] J.-P. Gauthier and V. Zakalyukin, On the motion planning problem, complexity, entropy, and nonholonomic interpolation. $J$. Dyn. Control Syst. 12 (2006) 371-404.

[24] H. Hermes, Nilpotent and high-order approximations of vector field systems. SIAM Rev. 33 (1991) 238-264.

[25] F. Jean, Complexity of nonholonomic motion planning. Int. J. Control 74 (2001) 776-782.

[26] F. Jean, Uniform estimation of sub-Riemannian balls. J. Dynam. Control Systems 7 (2001) 473-500.

[27] F. Jean, Entropy and complexity of a path in sub-Riemannian geometry. ESAIM: COCV 9 (2003) 485-508. 
[28] D. Jerison and A. Sánchez-Calle, Subelliptic, second order differential operators, in Complex analysis, III (College Park, Md., 1985-86). Vol. 1277 of Lect. Notes Math. Springer, Berlin (1987) 46-77.

[29] V. Jurdjevic, Geometric control theory. Vol. 52 of Cambridge Stud. Adv. Math. Cambridge University Press, Cambridge (1997).

[30] J. Mitchell, On Carnot-Carathéodory metrics. J. Differ. Geom. 21 35-45, 1985.

[31] C. Romero-Meléndez, J.P. Gauthier and F. Monroy-Pérez, On complexity and motion planning for co-rank one sub-Riemannian metrics. ESAIM: COCV 10 (2004) 634-655.

[32] L.P. Rothschild and E.M. Stein, Hypoelliptic differential operators and nilpotent groups. Acta Math. 137 (1976) $247-320$.

[33] J. San Martín, T. Takahashi and M. Tucsnak, A control theoretic approach to the swimming of microscopic organisms. Quart. Appl. Math. 65 (2007) 405-424.

[34] H.J. Sussmann, Some properties of vector field systems that are not altered by small perturbations. J. Differ. Equ. 20 (1976) $292-315$.

[35] H.J. Sussmann, Some recent results on the regularity of optimal cost functions, in Proc. of the Berkeley-Ames conference on nonlinear problems in control and fluid dynamics (Berkeley, Calif. (1983)), Lie Groups: Hist., Frontiers and Appl. Ser. B: Systems Inform. Control, II. Math Sci Press. Brookline, MA (1984) 429-434.

[36] H.J. Sussmann, A general theorem on local controllability. SIAM J. Control Optim. 25 (1987) 158-194.

[37] H.J. Sussmann, Regular synthesis for time-optimal control of single-input real analytic systems in the plane. SIAM J. Control Optim. 25 (1987) 1145-1162.

[38] E. Trélat, Some properties of the value function and its level sets for affine control systems with quadratic cost. J. Dyn. Control Systems 6 (2000) 511-541. 